\title{
CLASH: THE CONCENTRATION-MASS RELATION OF GALAXY CLUSTERS
}

\author{
J. Merten ${ }^{1,2,3}$, M. Meneghetti ${ }^{1,4,5}$, M. Postman ${ }^{6}$, K. Umetsu ${ }^{7}$, A. Zitrin ${ }^{2,32}$, E. Medezinski ${ }^{8}$, M. Nonino ${ }^{9}$, A. Koekemoer ${ }^{6}$, \\ P. Melchior ${ }^{10}$, D. Gruen ${ }^{11,12}$, L. A. Moustakas ${ }^{1}$, M. Bartelmann ${ }^{13}$, O. Host ${ }^{14}$, M. Donahue ${ }^{15}$, D. Coe ${ }^{6}$, A. Molino ${ }^{16}$, \\ S. Jouvel ${ }^{17,18}$, A. Monna ${ }^{11,12}$, S. Seitz ${ }^{11,12}$, N. Czakon ${ }^{7}$, D. Lemze ${ }^{8}$, J. SAyers ${ }^{2}$, I. Balestra ${ }^{9,19}$, P. Rosati ${ }^{20}$, N. Benítez ${ }^{15}$, \\ A. Biviano 9 , R. Bouwens ${ }^{21}$, L. Bradley ${ }^{6}$, T. Broadhurst ${ }^{22,23}$, M. Carrasco ${ }^{13,24}$, H. Ford ${ }^{8}$, C. Grillo $^{14}$, L. Infante $^{25}$, \\ D. Kelson ${ }^{26}$, O. Lahav ${ }^{17}$, R. Massey ${ }^{27}$, J. Moustakas ${ }^{28}$, E. Rasia ${ }^{29}$, J. Rhodes ${ }^{1,2}$, J. VeGA ${ }^{30,31}$, AND W. ZhenG ${ }^{6}$ \\ ${ }^{1}$ Jet Propulsion Laboratory, California Institute of Technology, 4800 Oak Grove Drive, Pasadena, CA 91109, USA; jmerten@ caltech.edu \\ ${ }^{2}$ California Institute of Technology, MC 249-17, Pasadena, CA 91125, USA \\ ${ }^{3}$ Department of Physics, University of Oxford, Keble Road, Oxford OX1 3RH, UK \\ ${ }^{4}$ INAF, Osservatorio Astronomico di Bologna, via Ranzani 1, I-40127 Bologna, Italy \\ 5 INFN, Sezione di Bologna, Viale Berti Pichat 6/2, I-40127 Bologna, Italy \\ ${ }^{6}$ Space Telescope Science Institute, 3700 San Martin Drive, Baltimore, MD 21208, USA \\ ${ }^{7}$ Institute of Astronomy and Astrophysics, Academia Sinica, P.O. Box 23-141, Taipei 10617, Taiwan \\ ${ }^{8}$ Department of Physics and Astronomy, The Johns Hopkins University, 3400 North Charles Street, Baltimore, MD 21218, USA \\ ${ }_{9}$ INAF/Osservatorio Astronomico di Trieste, via G.B. Tiepolo 11, I-34143 Trieste, Italy \\ ${ }^{10}$ Center for Cosmology and Astro-Particle Physics and Department of Physics, The Ohio State University, Columbus, OH 43210, USA \\ ${ }^{12}$ Max-Planck-Institute für extraterrestrische Physik, Giessenbachstr. 1, D-85748 Garching, Germany \\ ${ }^{13}$ Universität Heidelberg, Zentrum für Astronomie, Institut für Theoretische Astrophysik, Philosophenweg 12, D-69120 Heidelberg, Germany \\ ${ }^{14}$ Dark Cosmology Centre, Niels Bohr Institute, University of Copenhagen, Juliane Maries Vej 30, DK-2100 Copenhagen, Denmark \\ ${ }^{5}$ Department of Physics and Astronomy, Michigan State University, East Lansing, MI 48824, USA \\ ${ }^{16}$ Instituto de Astrofísica de Andalucía (CSIC), E-18080 Granada, Spain \\ ${ }^{17}$ Institut de Ciéncies de l'Espai (IEEC-CSIC), E-08193 Bellaterra (Barcelona), Spain \\ ${ }_{18}^{18}$ Department of Physics and Astronomy, University College London, London WC1E 6BT, UK \\ ${ }^{19}$ INAF-Osservatorio Astronomico di Capodimonte, Via Moiariello 16, I-80131 Napoli, Italy \\ ${ }^{20}$ Dipartimento di Fisica e Scienze della Terra, Università degli Studi di Ferrara, Via Saragat 1, I-44122 Ferrara, Italy \\ ${ }^{21}$ Leiden Observatory, Leiden University, P. O. Box 9513, NL-2333 Leiden, The Netherlands \\ 22 Department of Theoretical Physics and History of Science, University of the Basque Country UPV/EHU, P.O. Box 644, E-48080 Bilbao, Spain \\ ${ }^{23}$ Ikerbasque, Basque Foundation for Science, Alameda Urquijo, 36-5 Plaza Bizkaia, E-48011 Bilbao, Spain \\ ${ }^{24}$ Instituto de Astrofsica, Facultad de Física, Pontificia Universidad Católica de Chile, Casilla 306, Santiago 22, Chile \\ ${ }^{25}$ Centro de Astro-Ingeniería, Departamento de Astronomía y Astrofísica, Pontificia Universidad Catolica de Chile, V. Mackenna 4860, Santiago, Chile \\ ${ }^{26}$ Observatories of the Carnegie Institution of Washington, Pasadena, CA 91101, USA \\ ${ }^{27}$ Institute for Computational Cosmology, Durham University, South Road, Durham DH1 3LE, UK \\ ${ }^{28}$ Department of Physics and Astronomy, Siena College, 515 Loudon Road, Loudonville, NY 12211, USA \\ ${ }^{29}$ Physics Dept., University of Michigan, 450 Church Ave, Ann Arbor, MI 48109, USA \\ ${ }^{30}$ Departamento de Física Teórica, Universidad Autónoma de Madrid, Cantoblanco, E-28049 Madrid, Spain \\ ${ }^{31}$ LERMA, CNRS UMR 8112, Observatoire de Paris, 61 Avenue de l'Observatoire, F-75014 Paris, France \\ Received 2014 April 8; accepted 2015 April 2; published 2015 June 3
}

\begin{abstract}
We present a new determination of the concentration-mass ( $\mathrm{c}-\mathrm{M})$ relation for galaxy clusters based on our comprehensive lensing analysis of $19 \mathrm{X}$-ray selected galaxy clusters from the Cluster Lensing and Supernova Survey with Hubble (CLASH). Our sample spans a redshift range between 0.19 and 0.89 . We combine weaklensing constraints from the Hubble Space Telescope (HST) and from ground-based wide-field data with strong lensing constraints from HST. The results are reconstructions of the surface-mass density for all CLASH clusters on multi-scale grids. Our derivation of Navarro-Frenk-White parameters yields virial masses between $0.53 \times 10^{15} M_{\odot} / h$ and $1.76 \times 10^{15} M_{\odot} / h$ and the halo concentrations are distributed around $c_{200 c} \sim 3.7$ with a $1 \sigma$ significant negative slope with cluster mass. We find an excellent $4 \%$ agreement in the median ratio of our measured concentrations for each cluster and the respective expectation from numerical simulations after accounting for the CLASH selection function based on X-ray morphology. The simulations are analyzed in two dimensions to account for possible biases in the lensing reconstructions due to projection effects. The theoretical c$\mathrm{M}$ relation from our X-ray selected set of simulated clusters and the $\mathrm{c}-\mathrm{M}$ relation derived directly from the CLASH data agree at the $90 \%$ confidence level.
\end{abstract}

Key words: dark matter - galaxies: clusters: general - gravitational lensing: strong - gravitational lensing: weak

\section{INTRODUCTION}

The standard model of cosmology $(\Lambda \mathrm{CDM})$ is extremely successful in explaining the observed large-scale structure of the universe (see, e.g., Anderson et al. 2012; Planck Collaboration et al. 2014). However, when moving to progressively smaller length scales, inconsistencies between

\footnotetext{
${ }^{32}$ Hubble Fellow.
}

theoretical predictions and real observations have emerged. Examples include the cored mass-density profiles of dwarfspheroidal galaxies (Walker \& Peñarrubia 2011), the abundance of Milky Way satellites (Boylan-Kolchin et al. 2012), and the flat dark matter density profiles in the cores of galaxy clusters (Sand et al. 2002; Newman et al. 2013).

Galaxy clusters are unique tracers of cosmological structure formation (e.g., Voit 2005; Borgani \& Kravtsov 2011). As the 
largest collapsed objects in the observable universe, clusters form the bridge between the large-scale structure of the universe and the astrophysical regime of individual halos. From an observational point of view, all main mass components of a cluster, hot ionized gas, dark matter, and luminous stars, are directly or indirectly observable with the help of X-ray observatories (e.g., Rosati et al. 2002; Ettori et al. 2013), gravitational lensing (e.g., Bartelmann \& Schneider 2001; Bartelmann 2010), or optical observations.

As shown by numerical simulations (Navarro et al. 1996), dark matter tends to arrange itself following a specific, spherically symmetric density profile

$$
\rho_{\mathrm{NFW}}(r)=\frac{\rho_{s}}{r / r_{s}\left(1+r / r_{s}\right)^{2}},
$$

where the only two parameters $\rho_{s}$ and $r_{s}$ are a scale density and a scale radius. This functional form is now commonly called the Navarro-Frenk-White (NFW) density profile. It was found to fit well the dark matter distribution of halos in numerical simulations, independent of halo mass, cosmological parameters, or formation time (Navarro et al. 1997; Bullock et al. 2001).

A specific parametrization of the NFW profile uses the total mass enclosed within a certain radius $r_{\Delta}$

$$
M_{\Delta}=4 \pi \rho_{s} r_{s}^{3}\left(\ln \left(1+c_{\Delta}\right)-\frac{c_{\Delta}}{1+c_{\Delta}}\right)
$$

and the concentration parameter

$$
c_{\Delta}=\frac{r_{\Delta}}{r_{s}} .
$$

When applying the relations above to a specific analysis, the radius $r_{\Delta}$ is chosen such that it describes the halo on the scale of interest. An example is the radius at which the average density of the halo is 200 times the critical density of the universe at this redshift $(\Delta=200 c)$. Cosmological simulations show that there is a correlation between mass and concentration for dark matter structures, although with significant scatter. This defines the concentration-mass (c-M) relation which is a mild function of formation redshift and halo mass (Bullock et al. 2001; Eke et al. 2001; Zhao et al. 2003; Duffy et al. 2008; Gao et al. 2008; Klypin et al. 2011; Prada et al. 2012; Bhattacharya et al. 2013).

Observational efforts have been undertaken to measure the c-M relation either using gravitational lensing (Comerford \& Natarajan 2007; Oguri et al. 2012; Okabe et al. 2013), X-ray observations (Buote et al. 2007; Schmidt \& Allen 2007; Ettori et al. 2010), or dynamical analysis of cluster members (Lemze et al. 2009; Wojtak \& Łokas 2010; Biviano et al. 2013). Some of the observed relations are in tension with the predictions of numerical simulations (Duffy et al. 2008; Fedeli 2012). The most prominent example of such tension is the cluster Abell 1689 (Broadhurst et al. 2005; Peng et al. 2009, and references therein), with a concentration parameter up to a factor of three higher than predicted. In a follow-up study, Broadhurst et al. (2008) compared a larger sample of five clusters to the prediction from $\Lambda \mathrm{CDM}$ and found the derived $\mathrm{c}-\mathrm{M}$ relation in tension with the theoretical expectations (see also Broadhurst \& Barkana 2008a; Zitrin et al. 2010; Meneghetti et al. 2011). Possible explanations for these discrepancies include a selection-bias of the cluster sample since these clusters were known strong lenses, paired with the assumption of spherical symmetry for these systems (Hennawi et al. 2007; Meneghetti et al. 2010a). Moreover, the influence of baryons on the cluster core (Fedeli 2012; Killedar et al. 2012) and even the effects of early dark energy (Fedeli \& Bartelmann 2007; Sadeh \& Rephaeli 2008; Francis et al. 2009; Grossi \& Springel 2009) have been introduced as possible explanations. Ultimately, a new set of high-quality observations of an unbiased ensemble of clusters was needed to answer the question if observed galaxy clusters are indeed in tension with our cosmological standard model.

The Cluster Lensing And Supernova Survey with Hubble (CLASH; Postman et al. 2012a) is a multi-cycle treasury program, using 524 Hubble Space Telescope (HST) orbits to target 25 galaxy clusters, largely drawn from the Abell and MACS cluster catalogs (Abell 1958; Abell et al. 1989; Ebeling et al. 2001, 2007, 2010). Twenty clusters were specifically selected by their largely unperturbed X-ray morphology with the goal of representing a sample of clusters with regular, unbiased density profiles that allow for an optimal comparison to models of cosmological structure formation. As reported in Postman et al. (2012a) all clusters of the sample are fairly Xray luminous with X-ray temperatures $T_{x} \geqslant 5 \mathrm{keV}$ and show a smooth morphology in their X-ray surface brightness. For all systems the separation between the brightest cluster galaxy (BCG) and the X-ray luminosity centroid is $<20 \mathrm{kpc}$. An overview of the basic properties of the sample can be found in Table 1. In the following we will use these X-ray selected clusters to derive the observed $\mathrm{c}-\mathrm{M}$ relation for CLASH clusters based on weak and strong lensing and perform a thorough comparison to the theoretical expectation from numerical simulations. This study has two companion papers. The weak-lensing and magnification analysis of CLASH clusters by Umetsu et al. (2014) and the detailed characterization of numerical simulations of CLASH clusters by Meneghetti et al. (2014).

This paper is structured as follows. Section 2 provides a basic introduction to gravitational lensing and introduces the method used to recover the dark matter distribution from the observational data. The respective input data is described in Section 3 and the resulting mass maps and density profiles of the CLASH clusters are presented in Section 4. We interpret our results by a detailed comparison to theoretical c-M relations from the literature in Section 5 and use our own tailored set of simulations to derive a CLASH-like c-M relation in Section 6. We conclude in Section 7. Throughout this work we assume a flat cosmological model similar to a WMAP7 cosmology (Komatsu et al. 2011) with $\Omega_{m}=0.27, \Omega_{\Lambda}=0.73$, and a Hubble constant of $h=0.7$. For the redshift range of our cluster sample this translates to physical distance scales of $3.156-7.897 \mathrm{kpc} / \prime$.

\section{CLUSTER MASS PROFILES FROM GRAVITATIONAL LENSING}

We use gravitational lensing to recover the distribution of matter in galaxy clusters from imaging data. Lensing is particularly well-suited for this purpose since it is sensitive to the lens' total matter content, independent of its composition and under a minimum number of assumptions. After we discussed the basics of this powerful technique we will present a non-parametric inversion algorithm which maps the dark matter mass distribution over a wide range of angular scales. 
Table 1

The CLASH X-Ray Selected Cluster Sample

\begin{tabular}{|c|c|c|c|c|c|c|}
\hline Name & $z$ & $\begin{array}{c}\text { R.A. } \\
(\mathrm{deg} / \mathrm{J} 2000)\end{array}$ & $\begin{array}{c}\text { Decl. } \\
(\operatorname{deg} / \mathrm{J} 2000)\end{array}$ & $\begin{array}{l}\mathrm{k} T_{x}^{\mathrm{a}} \\
(\mathrm{keV})\end{array}$ & $\begin{array}{c}L_{\mathrm{bol}}^{\mathrm{a}} \\
\left(10^{44} \mathrm{erg} \mathrm{s}^{-1}\right)\end{array}$ & $" \rightarrow \mathrm{kpc}^{\mathrm{b}}$ \\
\hline Abell 383 & 0.188 & 42.014090 & -3.5292641 & 6.5 & 6.7 & 3.156 \\
\hline Abell 209 & 0.206 & 22.968952 & -13.611272 & 7.3 & 12.7 & 3.392 \\
\hline Abell 1423 & 0.213 & 179.32234 & 33.610973 & 7.1 & 7.8 & 3.482 \\
\hline Abell 2261 & 0.225 & 260.61336 & 32.132465 & 7.6 & 18.0 & 3.632 \\
\hline RX J2129+0005 & 0.234 & 322.41649 & 0.0892232 & 5.8 & 11.4 & 3.742 \\
\hline Abell 611 & 0.288 & 120.23674 & 36.056565 & 7.9 & 11.7 & 4.357 \\
\hline MS 2137-2353 & 0.313 & 325.06313 & -23.661136 & 5.9 & 9.9 & 4.617 \\
\hline RXC J2248-4431 & 0.348 & 342.18322 & -44.530908 & 12.4 & 69.5 & 4.959 \\
\hline MACS J1115+0129 & 0.352 & 168.96627 & 1.4986116 & 8.0 & 21.1 & 4.996 \\
\hline MACS J1931-26 & 0.352 & 292.95608 & -26.575857 & 6.7 & 20.9 & 4.996 \\
\hline RX J1532.8+3021 & 0.363 & 233.22410 & 30.349844 & 5.5 & 20.5 & 4.931 \\
\hline MACS J1720+3536 & 0.391 & 260.06980 & 35.607266 & 6.6 & 13.3 & 5.343 \\
\hline MACS J0429-02 & 0.399 & 67.400028 & -2.8852066 & 6.0 & 11.2 & 5.411 \\
\hline MACS J1206-08 & 0.439 & 181.55065 & -8.8009395 & 10.8 & 43.0 & 5.732 \\
\hline MACS J0329-02 & 0.450 & 52.423199 & -2.1962279 & 8.0 & 17.0 & 5.815 \\
\hline RX J1347-1145 & 0.451 & 206.87756 & -11.752610 & 15.5 & 90.8 & 5.822 \\
\hline MACS J1311-03 & 0.494 & 197.75751 & -3.1777029 & 5.9 & 9.4 & 6.128 \\
\hline MACS J1423+24 & 0.545 & 215.94949 & 24.078459 & 6.5 & 14.5 & 6.455 \\
\hline MACS J0744+39 & 0.686 & 116.22000 & 39.457408 & 8.9 & 29.1 & 7.186 \\
\hline CL J1226+3332 & 0.890 & 186.74270 & 33.546834 & 13.8 & 34.4 & 7.897 \\
\hline
\end{tabular}

${ }^{\mathrm{a}}$ From Postman et al. (2012a) and references therein.

b Conversion factor to convert arcseconds to kpc at the cluster's redshift and given the cosmological background model.

The CLASH data were designed to provide a unique combination of angular resolution, depth and multi-wavelength coverage that allows many new multiply lensed galaxies to be identified and their redshifts to be accurately estimated. These data are ideal for use with the SaWLens algorithm, which makes no a priori assumptions about the distribution of matter in a galaxy cluster.

\subsection{Gravitational Lensing}

Gravitational lensing is a direct consequence of Einstein's theory of general relativity (see, e.g., Bartelmann 2010, for a complete derivation). For cluster-sized lenses the lens mapping can be described by the lens equation

$$
\boldsymbol{\beta}=\boldsymbol{\theta}-\boldsymbol{\alpha}(\boldsymbol{\theta}) .
$$

This lens equation describes how the original 2D angular position in the source plane $\boldsymbol{\beta}=\left(\beta_{1}, \beta_{2}\right)$ is shifted by a deflection angle $\boldsymbol{\alpha}=\left(\alpha_{1}, \alpha_{2}\right)$ to the angular coordinates $\boldsymbol{\theta}=\left(\theta_{1}, \theta_{2}\right)$ in the lens plane. From now on we denote the angular diameter distance between observer and lens as $D_{1}$, between observer and source as $D_{s}$, and between lens and source as $D_{\mathrm{ls}}$. The deflection angle depends on the surfacemass density distribution of the lens $\Sigma\left(D_{d} \boldsymbol{\theta}\right)$ and can be related to a lensing potential

$$
\psi(\boldsymbol{\theta}):=\frac{1}{\pi} \int d^{2} \theta^{\prime} \frac{\Sigma\left(D_{1} \boldsymbol{\theta}\right)}{\Sigma_{\mathrm{cr}}} \ln \left|\boldsymbol{\theta}-\boldsymbol{\theta}^{\prime}\right|
$$

which is a line of sight projected and rescaled version of the Newtonian potential. The cosmological background model enters this equation through the critical surface mass density for lensing given by

$$
\Sigma_{\mathrm{cr}}=\frac{c^{2}}{4 \pi G} \frac{D_{\mathrm{s}}}{D_{1} D_{\mathrm{ls}}},
$$

where $c$ is the speed of light and $G$ is Newton's constant. By introducing the complex lensing operators (e.g., Bacon et al. 2006; Schneider \& Er 2008) $\partial:=\left(\frac{\partial}{\partial \theta_{1}}+i \frac{\partial}{\partial \theta_{2}}\right)$ and $\partial^{*}:=\left(\frac{\partial}{\partial \theta_{1}}-i \frac{\partial}{\partial \theta_{2}}\right)$ one can derive important lensing quantities as derivatives of the lensing potential

$$
\begin{aligned}
& \alpha:=\partial \psi \quad s=1 \\
& 2 \gamma:=\partial \partial \psi \quad s=2 \\
& 2 \kappa:=\partial \partial^{*} \psi s=0
\end{aligned}
$$

where $\alpha$ is the complex form of the already known deflection angle, $\gamma$ is called the complex shear, and the scalar quantity $\kappa$ is called convergence. The behavior of each quantity under rotations of the coordinate frame is given by the spinparameter $s$.

When relating these basic lens quantities to observables one distinguishes two specific regimes. In the case of weak lensing the distortions induced by the lens mapping are small and due to the intrinsic ellipticity of galaxies, localized averages over an ensemble of sources are used to separate the lensing signal from the random orientation caused by the intrinsic ellipticity. These local averages of ellipticity measurements can be related to Equation (7) by the reduced shear $g$

$$
\langle\epsilon\rangle=g:=\frac{\gamma}{1-\kappa},
$$


where we defined the ellipticity of a galaxy as $|\epsilon|:=\frac{a-b}{a+b}$ with the two axes of the ellipse fulfilling $a>b$. This relation between the measured ellipticities and the properties applies only in the regime where $|g|<1$ and assumes that the shear is constant across a galaxy (Schneider \& Er 2008). To mitigate this, we exclude shear measurements inside the region where $|g|>1$ (see also Section 3.2). For the combination of galaxy shape moments used in the RRG method, the constant shear approximation remains correct (to within $1 \%$ for a singular isothermal sphere lens) outside 1.07 times the Einstein radius (Massey \& Goldberg 2008). This potential source of bias is far smaller than other sources of statistical error in our current analysis. For completeness we note that for $|g|>1$, the relation between the measured ellipticities and the properties of the lens switches to

$$
\langle\epsilon\rangle=\frac{1-\kappa}{\gamma^{*}} .
$$

For a more thorough description of the relation between the measured shapes of galaxy images and the lens properties in the weak lensing regime we refer to the review by (Bartelmann \& Schneider 2001, and references therein). We also do not discuss here the many systematic effects to be taken into account during such a shape measurement but refer to, e.g., Kitching et al. (2012), Massey et al. (2013), or Mandelbaum et al. (2014).

In the strong lensing regime, close to the core of the lens' mass distribution, the assumption of small image distortions does not hold any more. The lens equation becomes nonlinear and therefore multiple images of the same source can form. This happens near the critical line at a given redshift which is defined by the roots of the lensing Jacobian

$$
\operatorname{det} \mathcal{A}=(1-\kappa)^{2}-\gamma^{2} .
$$

While the weak lensing regime expands over the full cluster field, it does not describe the mass distribution in the center of the cluster. Strong lensing is limited to the inner-most $10^{\prime \prime}-50^{\prime \prime}$ of the cluster field, which renders the combination of the two regimes the ideal approach for mass reconstruction. One particular limitation of gravitational is the mass-sheet-degeneracy (Falco et al. 1985; Gorenstein et al. 1988). It describes the invariance of many lensing observables under the transformation

$$
\begin{gathered}
\kappa(\boldsymbol{\theta}) \rightarrow \kappa^{\prime}(\boldsymbol{\theta})=\lambda \kappa(\boldsymbol{\theta})+(1-\lambda) \\
\gamma(\boldsymbol{\theta}) \rightarrow \gamma^{\prime}(\boldsymbol{\theta})=\lambda \gamma(\boldsymbol{\theta})
\end{gathered}
$$

with the free ${ }^{33}$ transformation parameter $\lambda$. Several ways have been suggested to break the mass-sheet-degeneracy, including the use of magnification constraints (e.g., Broadhurst et al. 1995), which are not invariant under the mass-sheet transformation, and the inclusion of multi-redshift stronglensing features (Bradač et al. 2004, 2005b). In this work, however, we will folow another route to break the mass-sheetdegeneracy, which is the simple condition that the average

\footnotetext{
33 The parameter is free up to the limit that the solution for the surface-mass density must be physical in terms of e.g., the dynamics of cluster member galaxies etc.
}

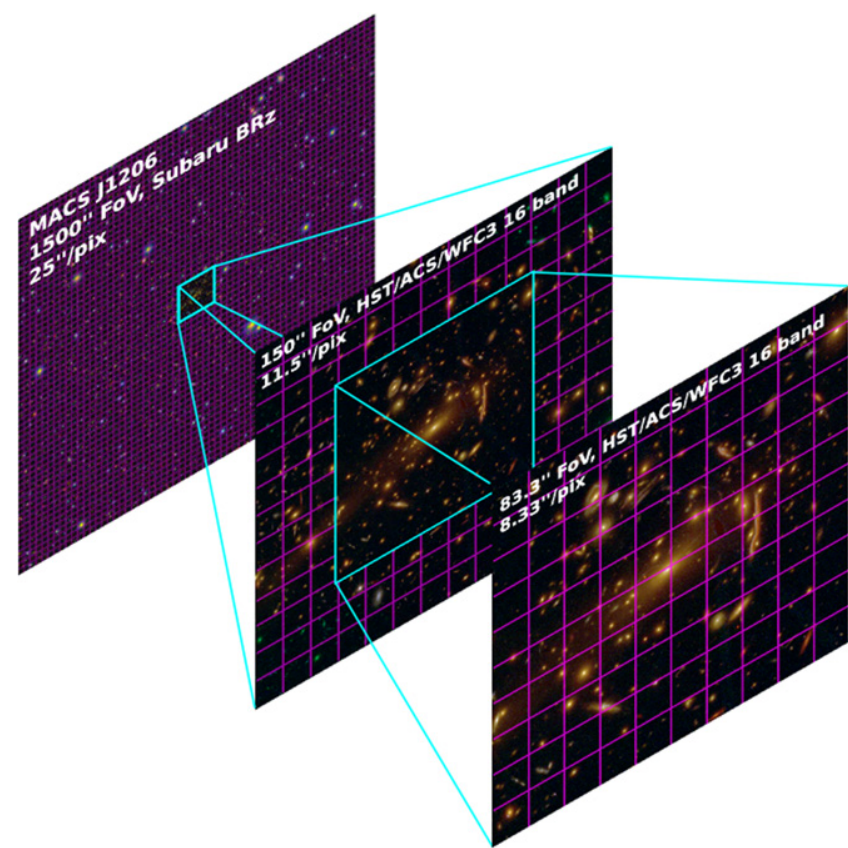

Figure 1. Visualization of our multi-scale approach. While weak lensing data from Subaru allows for a mass reconstruction of a galaxy cluster on a wide field, the achievable resolution is rather low. HST weak lensing delivers higher resolution but on a relatively small field. Finally, the strong lensing regime provides a very high resolution, but only in the inner-most cluster core. This figure shows one of our sample clusters, MACS J1206 and the reconstruction grids for all three lensing regimes.

convergence at the edge of the reconstruction field goes to zero in the absence of a lensing signal. This assumption is justified once wide-field imaging is used, entailing the full cluster field well-beyond its virial radius and we will present its specific implementation into our reconstruction algorithm in the next section.

\subsection{Non-parametric Lensing Inversion With SaWLens}

The SaWLens (Strong -and Weak-lensing) method was developed with two goals in mind. First, it should consistently combine weak and strong lensing. The second goal was to make no a priori assumptions about the underlying mass distribution, but to build solely upon the input data. The initial idea for such a reconstruction algorithm was formulated by Bartelmann et al. (1996) and was further developed by Seitz et al. (1998) and Cacciato et al. (2006). Similar ideas were implemented by Bradač et al. (2005b) with first applications to observations in Bradač et al. (2005a, 2006). Other nonparametric reconstruction algorithms, based on different methdologies, have been presented by Abdelsalam et al. (1998), Bridle et al. (1998), Liesenborgs et al. (2006), Jee et al. (2007), Diego et al. (2007), and Merten (2014). In its current implementation (Merten et al. 2009), SaWLens performs a reconstruction of the lensing potential (Equation (5)) on an adaptively refined grid. In this particular study, the method uses three different grid sizes to account for weak lensing on a wide field, such as is provided by ground-based telescopes, weak lensing constraints from the HST on a much smaller field of view but with considerably higher spatial resolution, and a fine grained grid to trace strong lensing features near the inner-most core of the cluster. This three-level adaptive grid is illustrated in Figure 1. 
SaWLens uses a statistical approach to reconstruct the lensing potential $\psi$ in every pixel of the grid. A $\chi^{2}$-function, which depends on the lensing potential and includes a weak and a strong-lensing term is defined by

$$
\chi^{2}(\psi)=\chi_{w}^{2}(\psi)+\chi_{s}^{2}(\psi),
$$

and the algorithm minimizes it such that the input data is best described by a pixelized lensing potential $\psi_{l}$

$$
\frac{\partial \chi^{2}(\psi)}{\partial \psi_{l}} \stackrel{!}{=} 0 \text {. }
$$

In Equation (14), $l$ runs over all grid pixels. The weak-lensing term in Equation (13) is derived from Equation (8) with a measured average complex ellipticity of background sources in each grid pixel $\epsilon$

$$
\chi_{\mathrm{w}}^{2}=\sum_{i, j}(\varepsilon-g(\psi))_{i} C_{i j}^{-1}(\varepsilon-g(\psi))_{j} .
$$

The covariance matrix $\mathcal{C}$ is non-diagonal because the algorithm adaptively averages over a number of background-ellipticity measurements in each pixel to account for the intrinsic ellipticity of background sources. Depending on the reconstruction resolution, this number is either defined by all weaklensing background galaxies that are contained within the area of the current reconstruction pixel, or, if the reconstruction resolution is high, the algorithm searches in progressively larger squares around the center of the reconstruction pixel until at least 10 galaxies are contained in the square area. Due to this averaging scheme, neighboring pixels may share a certain number of background sources and the algorithm keeps track of these correlations between pixels as described in Section 3.2 and especially Equations (14)-(16) of Merten et al. (2009). We do not perform any distance-weighting in our averages since we treat our reconstruction cells as extended square pixels. However, during the averaging process each measured ellipticity is weighted with the inverse-variance of the shape measurement. This approach has been calibrated and is tested by reconstructing numerically simulated lenses in Merten et al. (2009) and Meneghetti et al. (2010b). The connection to the lensing potential is given by Equation (8) which, when inserted into Equation (15), yields

$$
\begin{aligned}
\chi_{\mathrm{w}}^{2}(\psi)= & \sum_{i, j}\left(\varepsilon-\frac{Z(z) \gamma(\psi)}{1-Z(z) \kappa(\psi)}\right)_{i} \\
& \times \mathcal{C}_{i j}^{-1}\left(\varepsilon-\frac{Z(z) \gamma(\psi)}{1-Z(z) \kappa(\psi)}\right)_{j},
\end{aligned}
$$

where again both indices $i$ and $j$ run over all grid cells. Note that all lensing quantities given by Equation (7) have a redshift dependence introduced by the critical density in Equations (5) and (6). This is taken into account by a cosmological weight function (Bartelmann \& Schneider 2001) scaling each pixel to a fiducial redshift of infinity during the reconstruction.

$$
Z(z):=\frac{D_{\infty} D_{\mid s}}{D_{\mid \infty} D_{\mathrm{s}}} H\left(z-z_{\mid}\right) .
$$

The Heaviside step function ensures that only sources behind the lens redshift $z_{l}$ have non-zero weight.

The definition of the strong lensing term in Equation (13) makes use of the fact the position of the lens' critical line at a certain redshift can be inferred from the position of multiple images. It has been shown in Merten et al. (2009) and Meneghetti et al. (2010b) that pixel sizes $>5$ " are large enough to make this simple assumption. Therefore, following Equation (10)

$$
\begin{aligned}
\chi_{\mathrm{s}}^{2}(\psi) & =\frac{|\operatorname{det} \mathcal{A}(\psi)|_{i}^{2}}{\sigma_{i, \mathrm{~s}}^{2}} \\
& =\frac{\left.\left.\left|(1-Z(z) \kappa(\psi))^{2}-\right| Z(z) \gamma(\psi)\right|^{2}\right|_{i} ^{2}}{\sigma_{i, \mathrm{~s}}^{2}},
\end{aligned}
$$

where this term is only assigned to those grid cells which are part of the critical line at a certain redshift $z$ given the positions of multiple images. The error term $\sigma$ is then given by the cell size of the grid following

$$
\left.\sigma_{\mathrm{s}} \approx \frac{\partial \operatorname{det} \mathcal{A}}{\partial \theta}\right|_{\theta_{\mathrm{c}}} \delta \theta \approx \frac{\delta \theta}{\theta_{\mathrm{E}}},
$$

with $\theta_{\mathrm{E}}$ being an estimate of the Einstein radius of the lens.

The missing connection to the lensing potential $\psi$ is given by Equation (7). The numerical technique of finite differencing is then used to express the basic lensing quantities by simple matrix multiplications

$$
\begin{aligned}
\kappa_{i} & =\mathcal{K}_{i j} \psi_{j} \\
\gamma_{i}^{1} & =\mathcal{G}_{i j}^{1} \psi_{j} \\
\gamma_{i}^{2} & =\mathcal{G}_{i j}^{2} \psi_{j}
\end{aligned}
$$

where $\mathcal{K}_{i j}, \mathcal{G}_{i j}^{1}$, and $\mathcal{G}_{i j}^{2}$ are sparse matrices representing the finite differencing stamp of the respective differential operator (Seitz et al. 1998; Bradač et al. 2005a; Merten et al. 2009). With these identities in mind it can be shown that Equation (14) takes the form of a linear system of equations, which is solved numerically. There are two important aspects to this method, which we will only mention briefly. First, a two-level iteration scheme is employed to deal with the nonlinear nature of the reduced shear (Schneider \& Seitz 1995) and to avoid overfitting of local noise contributions (Merten et al. 2009). Second, a regularization scheme is adapted (Seitz et al. 1998; van Waerbeke 2000) to ensure a smooth transition from one iteration step to the next. In this work we adapt the regularization scheme of Bradač et al. (2005a), with an initial flat convergence prior, which regularizes the initial convergence to zero over the field. This also conveniently implements the way in which we break the mass-sheet-degeneracy, as we have mentioned earlier on. The initial regularization condition ensures a flat and zero convergence field where no significant lensing signal is found in the shear data.

It is important that a complex lensing inversion algorithm is tested thoroughly and under controlled but realistic conditions. Such tests are particular importance for our analysis since we are applying our method to a large set of real clusters of galaxies and we need to know our expected level of systematic error in the determination of masses and concentrations. Also, 
we use several techniques which rely on specific assumptions and hence need to be tested for their validity. This includes our way of breaking the mass-sheet degeneracy, the use of critical line estimators in the strong-lensing regime and the two-level iteration with a specific regularization scheme.

These tests were performed in Meneghetti et al. (2010b) with a set of three simulated clusters with masses between $6.8 \times 10^{14}-1.1 \times 10^{15} M_{\odot} / h$. Each of the three simluated clusters was reconstructed in three perpendicular projections, spanning a range of surface-mass densities of fairly round, to elliptical and highly substuctured morphologies. Figure 15 of Meneghetti et al. (2010b) shows that SaWLens determines the masses of this particular set of simulations with an accuracy of $5 \%-10 \%$ at all relevant radii. Other methods relying on either strong -or weak-lensing constraints are limited to either small or large scales and showed less accurate results with errors of $\sim 20 \%$. SaWLens also recovered the concentrations of the simulated halos with errors at the $\sim 5 \%$ level. These results on concentrations are summarized in Table 3 of Meneghetti et al. (2010b). We want to emphasize that the quoted errors refer to the results when reconstructing a set of nine projections from three cluster simulations. This number is smaller than the 19 cluster reconstructions shown in this work and the simulated cluster sample was also not explicitly constructed to mimick the CLASH selection. Hence, these tests can only serve as an approximate lead for the accuracy of individual cluster reconstructions of this work, but nevertheless represent an important check of our methodlogy and numerical implementation. Aside from the successful tests on simulated lenses, the SaWLens algorithm has been used in the reconstruction of observed galaxy clusters (Merten et al. 2009, 2011; Umetsu et al. 2012; Medezinski et al. 2013; Patel et al. 2014).

\section{THE CLASH DATA SET}

Our analysis focuses on the X-ray selected sub-sample of CLASH (Table 1). For each of these clusters a large number of lensing constraints was collected, either from the HST CLASH survey (Postman et al. 2012a), the accompanying Subaru/ Suprime-Cam (Umetsu et al. 2011; Postman et al. 2012a; Medezinski et al. 2013) or ESO/WFI (Gruen et al. 2013) weak lensing observations, or from the CLASH-Very Large Telescope (VLT) spectroscopic program (Balestra et al. 2013). The data collection includes strong-lensing multiply imaged systems together with accurate spectroscopic or photometric redshifts and weak-lensing shear catalogs on the full cluster field, paired with a reliable background selection of weak lensing sources.

\subsection{Strong Lensing in the HST Fields}

The Zitrin et al. (2009) method is applied to identify multiple-image systems in each cluster field. The respective strong-lensing mass models for several CLASH clusters have already been published (Zitrin et al. 2011, 2012a, 2012b, 2013; Coe et al. 2012, 2013; Umetsu et al. 2012; Zheng et al. 2012) and the full set of strong-lensing models and multiple-image identifications is presented in Zitrin et al. (2014). Exceptions are the cluster RXC J2248, where the multiple-image identification is based on the Monna et al. (2014) strong-lensing mass model, and RX J1532, where our team was not able to identify any strong-lensing features to date. In this case, we derive the underlying lensing potential from weak lensing only with a
Table 2

Strong-lensing Constraints

\begin{tabular}{lccccc}
\hline \hline Name & $N_{\text {sys }}{ }^{\mathrm{a}}$ & $N_{\text {spec }}{ }^{\mathrm{b}}$ & $N_{\text {crit }}{ }^{\mathrm{c}}$ & $z$-range & $\left\langle d_{\text {crit }}\right\rangle^{\mathrm{d}}$ \\
& & & & & $17.5 \pm 5.7$ \\
\hline Abell 383 & 9 & 5 & $20^{\mathrm{e}}$ & $1.01-6.03$ & $17.5 \pm 0.8$ \\
Abell 209 & 6 & 0 & 5 & $1.88-3.5$ & $8.5 \pm 0.5 \pm 1.2$ \\
Abell 1423 & 1 & 0 & 1 & 3.5 & $17.5 \pm-1.5$ \\
Abell 2261 & 12 & 0 & 18 & $1.54-4.92$ & $18.1 \pm 8.2$ \\
RX J2129+0005 & 4 & 1 & 8 & $0.55-1.965$ & $8.1 \pm 3.5$ \\
Abell 611 & 4 & 3 & 9 & $0.908-2.59$ & $13.1 \pm 4.5$ \\
MS 2137-2353 & 2 & 2 & 6 & $1.501-1.502$ & $12.2 \pm 4.7$ \\
RXC J2248-4431 & 11 & 10 & 22 & $1.0-6.0$ & $27.8 \pm 5.6$ \\
MACS J1115+0129 & 2 & 0 & 5 & $2.46-2.64$ & $19.9 \pm 9.2$ \\
MACS J1931-26 & 7 & 0 & 8 & $2.6-3.95$ & $29.2 \pm 1.3$ \\
RX J1532.8+3021 & 0 & 0 & 0 & $\ldots$ & $\ldots$ \\
MACS J1720+3536 & 7 & 0 & 11 & $0.6-4.6$ & $19.3 \pm 8.8$ \\
MACS J0429-02 & 3 & 0 & 6 & $1.6-4.1$ & $11.8 \pm 3.6$ \\
MACS J1206-08 & 13 & 4 & 33 & $1.033-5.44$ & $28.1 \pm 14.8$ \\
MACS J0329-02 & 6 & 0 & 12 & $1.55-6.18$ & $23.7 \pm 5.2$ \\
RX J1347-1145 & 13 & 1 & 15 & $0.7-4.27$ & $31.6 \pm 13.3$ \\
MACS J1311-03 & 2 & 0 & 4 & $2.63-6.0$ & $12.9 \pm 5.3$ \\
MACS J1423+24 & 5 & 3 & 18 & $1.779-2.84$ & $15.0 \pm 5.6$ \\
MACS J0744+39 & 5 & 0 & 8 & $1.15-4.62$ & $31.6 \pm 16.2$ \\
CL J1226+3332 & 4 & 0 & 9 & $2.0-4.2$ & $23.2 \pm 12.2$ \\
\hline
\end{tabular}

${ }^{a}$ The number of multiple-image systems in this cluster field.

b The number of spectroscopically confirmed multiple-image systems.

${ }^{c}$ The number of critical line estimators derived from the position of multipleimage systems.

${ }^{\mathrm{d}}$ The mean distance and its standard deviation from the cluster center to the critical line estimators.

e An illustration of how the critical line estimators for this specific systems were derived is given in Figure 2.

significantly coarser resolution in the central region, compared to the strong-lensing clusters.

A summary of multiple-image systems found in each cluster is given in Table 2. From the identified multiple images we estimate the locations of critical lines following the approach of Merten et al. (2009). We show this critical line estimation for one concrete example in Figure 2, where we indicate the multiple images identified by Zitrin et al. (2011) in Abell 383 together with the critical lines derived from a detailed stronglensing model of the cluster. In addition we show our critical line estimation from the multiple-image identifications which is in excellent agreement with the critical lines from the stronglensing model given the pixel size of our reconstruction. It is not possible to determine the position of the critical line to high accurazy from multiple images only. In fact, only a conservative and coarse resolution in the strong-lensing regime of $5^{\prime \prime}-10^{\prime \prime}$ renders the positional error in the critical line estimation negligible when compared to the reconstruction resolution. We show this for a concrete example in Figure 2. However, we are not limited by this coarse resolution since we still map the density profile over three decades in radius and since we are not aiming to break the mass-sheet-degeneracy using multi-plane strong-lensing features, as, e.g., shown in Bradač et al. (2005b).

Redshifts for all strong lensing features are either taken from the literature, spectroscopic redshifts from the on-going CLASH VLT-Vimos large program (186.A-0798) (Balestra et al. 2013), or from the CLASH photometry directly using Bayesian photometric redshifts (BPZ, Benítez 2000). CLASH 


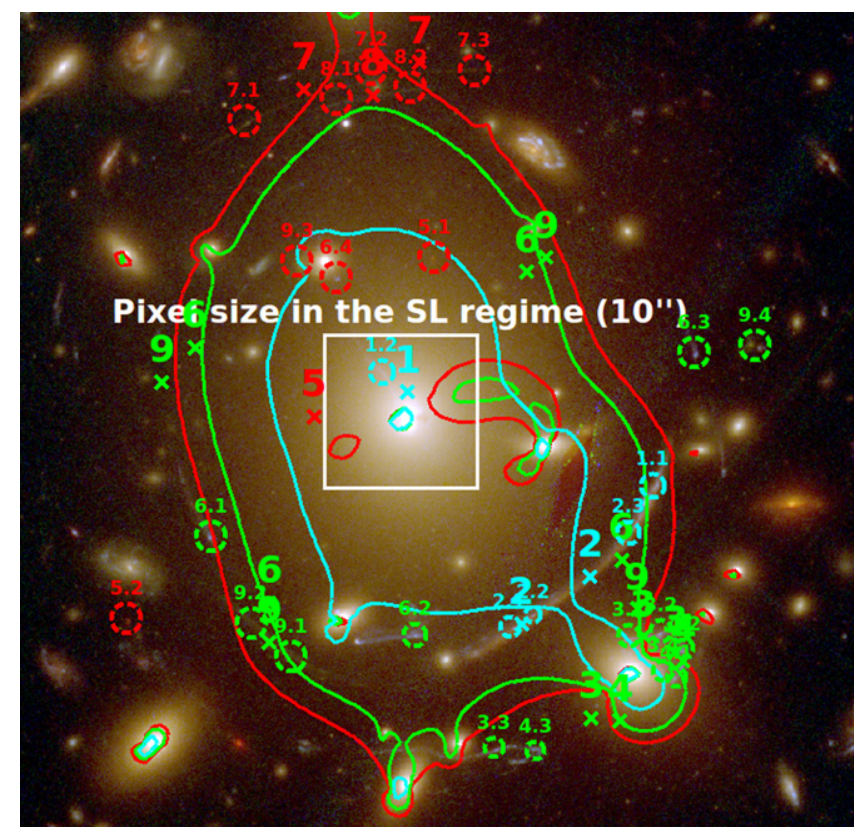

Figure 2. Estimation of the critical line for the SaWLens analysis of Abell 383. Shown by the labeled circles are the different sets of multiple-image systems identified by Zitrin et al. (2011). The three solid lines show the critical lines from their strong lensing model for three different source redshifts (cyan: $z_{s}=1.01$, green: $z_{s}=2.55$ and red: $z_{s}=6.03$ ). The crosses with integer labels show our critical line estimate for a particular multiple image system with the same ID number. The white box shows the SaWLens pixel size in the stronglensing regime. The critical line estimates and the multiple-image systems are divided into three groups. Cyan indicates systems at $z_{s}=1.01$, green contains systems in a redshift range between $z_{s}=2.20$ and $z_{s}=3.90$, and red systems in the range from $z_{s}=4.55$ to $z_{s}=6.03$

has been explicitly designed to deliver accurate photometric redshifts for strong lensing features (Postman et al. 2012a). The accuracy of the CLASH photometric redshifts has been recently evaluated in Jouvel et al. (2014) where we found $3.0 \%(1+z)$ precision for strong-lensing arcs and field galaxies.

\subsection{Weak Lensing in the HST Fields}

For cluster mass reconstruction, the HST delivers a four to five times higher density of weakly lensed background galaxies than observations from the ground (e.g., Clowe et al. 2006; Bradač et al. 2006, 2008; Merten et al. 2011; Jee et al. 2012). We measure the shapes of background galaxies in typically seven broad-band Advanced Camera for Surveys (ACS) filters, F435W, F475W, F606W, F625W, F775W, F814W, and F850LP. The full survey design is laid out in detail in Postman et al. (2012a). Where available, the CLASH data is augmented by archival HST observations. For the F814W and F850LP filters, two HST orbits are allocated for each CLASH cluster, which are split into four different visits with two different $H S T$ roll angles. The total exposure time in the other filters is one orbit which is split into two separate visits. Each single visit consists of two sequential, dithered expsures. Each of the individiual exposures is corrected for charge-transfer-inefficiency (e.g., Anderson \& Bedin 2010; Massey 2010; Jee et al. 2014a) by using the PixCteCorr routine in the STScI Python package. This procedure is based on the pixel-based correction algorithm proposed in Anderson \& Bedin (2010). In order to improve the spatial sampling of the PSF and to avoid hot pixels and detector imperfections we do not measure shapes in the individual expsures of each visit but combine the two exposures with a modified version of the MosaicDrizzle pipeline (Koekemoer et al. 2002, 2011) with a drizzle pixel scale of $0 . \prime 03$. This is possible since the two expsoures in each visit are taken sequentially and the time-dependent variation of the HST point-spread function (PSF) is small between the two exposures. In contrast, individual exposures of different visits might be separated by several days, which is why we do not work with the total coadd, based on all visits in a single filter. A final set of bad pixel and cosmic ray masks is provided by the MosaicDrizzle pipeline using all exposures in multiple epochs for a given filter as described in Postman et al. (2012a). For shape measurement and PSF correction we use the RRG package (Rhodes et al. 2000), which implements an HST breathing model (Leauthaud et al. 2007; Rhodes et al. 2007) to correct for the thermally induced variation of the HST PSF. The method has been used for cosmic shear (Massey et al. 2007) and cluster lensing (Bradač et al. 2008; Merten et al. 2011) applications following testing and calibration on shapelet-based image simulations (Massey et al. 2004) when it was implemented in the context of the COSMOS survey (see Figure 14 of Leauthaud et al. 2007). The shear calibration found an overall multiplicative bias of $1-0.86_{-0.05}^{+0.07}$ and RRG applies a correction factor accordingly. To be more precise, the two shear components are multiplied with a factor of $(0.80)^{-1}$ and $(0.92)^{-1}$ for the first and the second shear component, respectively, following the findings of Leauthaud et al. (2007). The additive and quadratic bias was found to be negligible (see Table 5 in Leauthaud et al. 2007). The level of PSF variation was determined from the inspection of stars in the field of each visit (Rhodes et al. 2007) and by cross-comparison with the STScI focus tool ${ }^{34}$ (di Nino et al. 2008, and references therein). For the shape measurements in each visit we discard all galaxies with signal-to-noise ratio $(\mathrm{S} / \mathrm{N})<10$ and every shear catalog is then rotated into a north-up orientation in order to have a unique orientation reference for the directional shape parameters. The individual visit catalogs are finally combined using a $\mathrm{S} / \mathrm{N}$ weighted average for multiple measurements of the same object. This procedure is applied to each of the seven ACS filters. Catalogs in different filters are combined by using a signal-to-noise weighted average for matching sources. In the case of Abell 611 we did not use F606W and F625W images since the focus tool did not cover the time period when these observations were taken. In the case of RX J2129 additional F555W data is included from archival data. We show the remaining residual PSF in Figure 3, where the two ellipticity components of bright un-saturated stars $(18 \lesssim \mathrm{F} 814 \mathrm{~W} \lesssim 22)$ in the exposures of all clusters and for different filter configurations are shown after PSF correction.

The lensed background sample for each combined catalog was selected using two photo- $z$ criteria. First, the most likely redshift according to the probability distribution of BPZ had to be at least $20 \%$ larger than the cluster redshift to ensure a limited contamination by cluster members. Second, the lower bound on the source redshift (based on the BPZ probability distribution) had to be larger or equal to the cluster redshift. A size cut and removal of obvious artifacts finalizes each $H S T$ weak lensing catalog and the effective lensing redshift of the background distribution is determined from the photometric redshift of each object in the final catalog. All relevant

\footnotetext{
${ }^{34}$ http://www.stsci.edu/hst/observatory/focus
} 

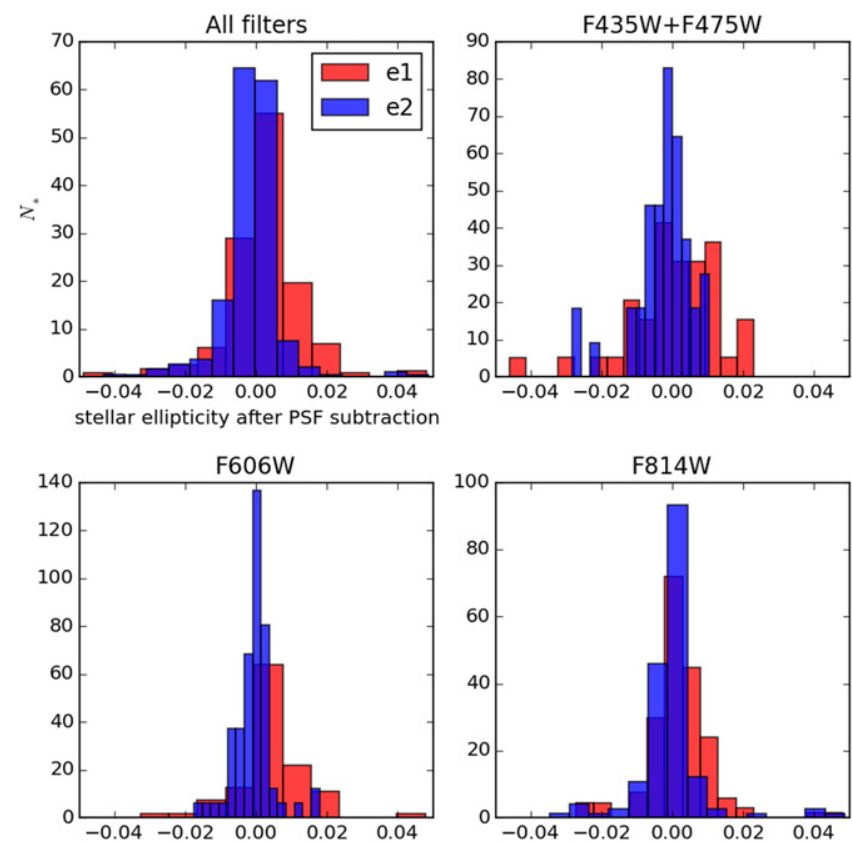

Figure 3. Residual stellar ellipticity after PSF correction with the RRG pipeline. The histograms show both ellipticity components of bright, unsaturated stars $(18 \lesssim \mathrm{F} 814 \mathrm{~W} \lesssim 22)$ in our ACS exposures of all sample clusters. The upper right panel shows the residual ellipticity distribution for a joint catalog using all filters, the other three panels show individual contributions for catalogs in specific filters as indicated by the panel titles.

information about the HST weak lensing catalogs is summarized in Table 3.

The cross-shear component was found to be small at all radii. To see this in the case of our HST weak lensing we refer to the panels for Abell 1423 and CL J1226 in Figure 5. We also found strong correlations in both ellipticity components between different ACS filter measurements. This is demonstrated for four different filters and four different clusters in Figure 4. As a final cross-check we performed lensing inversions of the HST weak lensing data only, as it is shown for the case of Abell 1423 in Figure 15; all of these showed strong correlations with the light distribution in the HST fields. Our selection of weak-lensing galaxies in the HST fields is finalized by discarding all galaxies which lie within the critical curve of the lens. While doing so, we ensure that Equation (8) holds for all measured reduced shear values in our reconstructed field and we justify this step with the fact that the strong-lensing regime of all our lenses is well-constrained by the stronglensing features in the field. We determine the position of the critical lines with the strong-lensing models presented in Zitrin et al. (2014).

\subsection{Weak Lensing in the Ground-based Fields}

The creation of our weak-lensing shear catalogs from ground-based observations is described in detail in Section 4 of Umetsu et al. (2014). For completeness we summarize the properties of these catalogs in Table 4 and list the main steps of our analysis in the following.

The wide-field weak-lensing pipeline of Umetsu et al. (2014) is implemented based on the PSF-correction and shearcalibration procedures outlined in (Umetsu et al. 2010, see Section 3.2) In the course of the CLASH survey, this analysis
Table 3

HST Weak-lensing Constraints

\begin{tabular}{lcccc}
\hline \hline Name & $N_{\text {band }}{ }^{\mathrm{a}}$ & $N_{\text {gal }}{ }^{\mathrm{b}}$ & $\begin{array}{c}\rho_{\text {gal }}^{\mathrm{c}} \\
\left(\mathrm{arcmin}^{-2}\right)\end{array}$ & $z_{\text {eff }}{ }^{\mathrm{d}}$ \\
\hline Abell 383 & 7 & 796 & 50.7 & 0.90 \\
Abell 209 & 7 & 832 & 44.0 & 0.95 \\
Abell 1423 & 7 & 807 & 50.3 & 0.92 \\
Abell 2261 & 7 & 725 & 46.7 & 0.79 \\
RX J2129+0005 & 8 & 624 & 35.8 & 0.82 \\
Abell 611 & 5 & 547 & 42.3 & 0.86 \\
MS 2137-2353 & 7 & 801 & 48.3 & 1.12 \\
RXC J2248-4431 & 7 & 598 & 38.5 & 1.12 \\
MACS J1115+0129 & 7 & 491 & 37.4 & 1.03 \\
MACS J1931-26 & 7 & 709 & 59.5 & 0.82 \\
RX J1532.8+3021 & 7 & 508 & 35.9 & 1.07 \\
MACS J1720+3536 & 7 & 635 & 40.6 & 1.11 \\
MACS J0429-02 & 7 & 654 & 42.4 & 1.08 \\
MACS J1206-08 & 7 & 581 & 51.2 & 1.13 \\
MACS J0329-02 & 7 & 493 & 35.2 & 1.18 \\
RX J1347-1145 & 7 & 633 & 45.7 & 1.13 \\
MACS J1311-03 & 7 & 447 & 33.7 & 1.03 \\
MACS J1423+24 & 7 & 899 & 75.3 & 1.04 \\
MACS J0744+39 & 7 & 743 & 61.3 & 1.32 \\
CL J1226+3332 & 7 & 925 & 32.7 & 1.66 \\
\hline
\end{tabular}

a The number of HST/ACS bands from which the final shear catalog was created.

$\mathrm{b}$ The number of background selected galaxies in the shear catalog.

c The surface-number density of background selected galaxies in the field.

${ }^{\mathrm{d}}$ The effective redshift of the background sample, derived from their photo- $z s$ and by calculating the average of the $D_{\mathrm{ls}} / D_{s}$ ratio and correcting for the nonlinearity of the reduced shear.
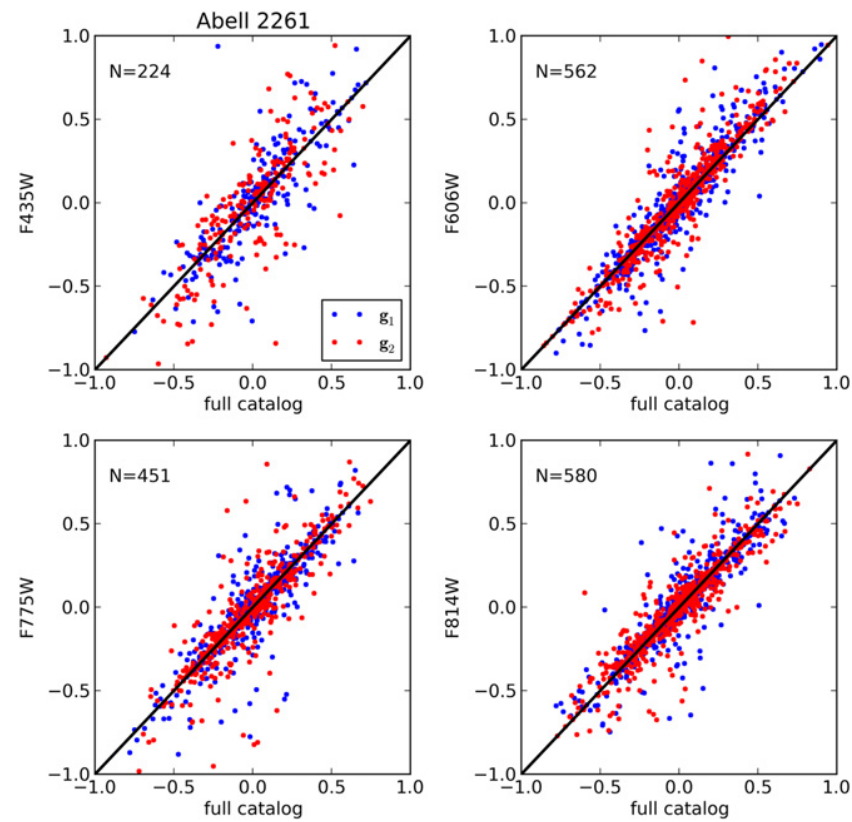

Figure 4. Correlation of shape measurements in different HST/ACS filters for the example of Abell 2261. The upper left panel shows the correlation of ellipticities measured in the F435W images compared to the combined HST/ ACS catalogs. The upper right, lower left, and lower right panels show the same correlation for the F606W, F775W, and F814W catalogs. Also shown in each individual plot is the number of overlapping galaxies in the different catalogs. 

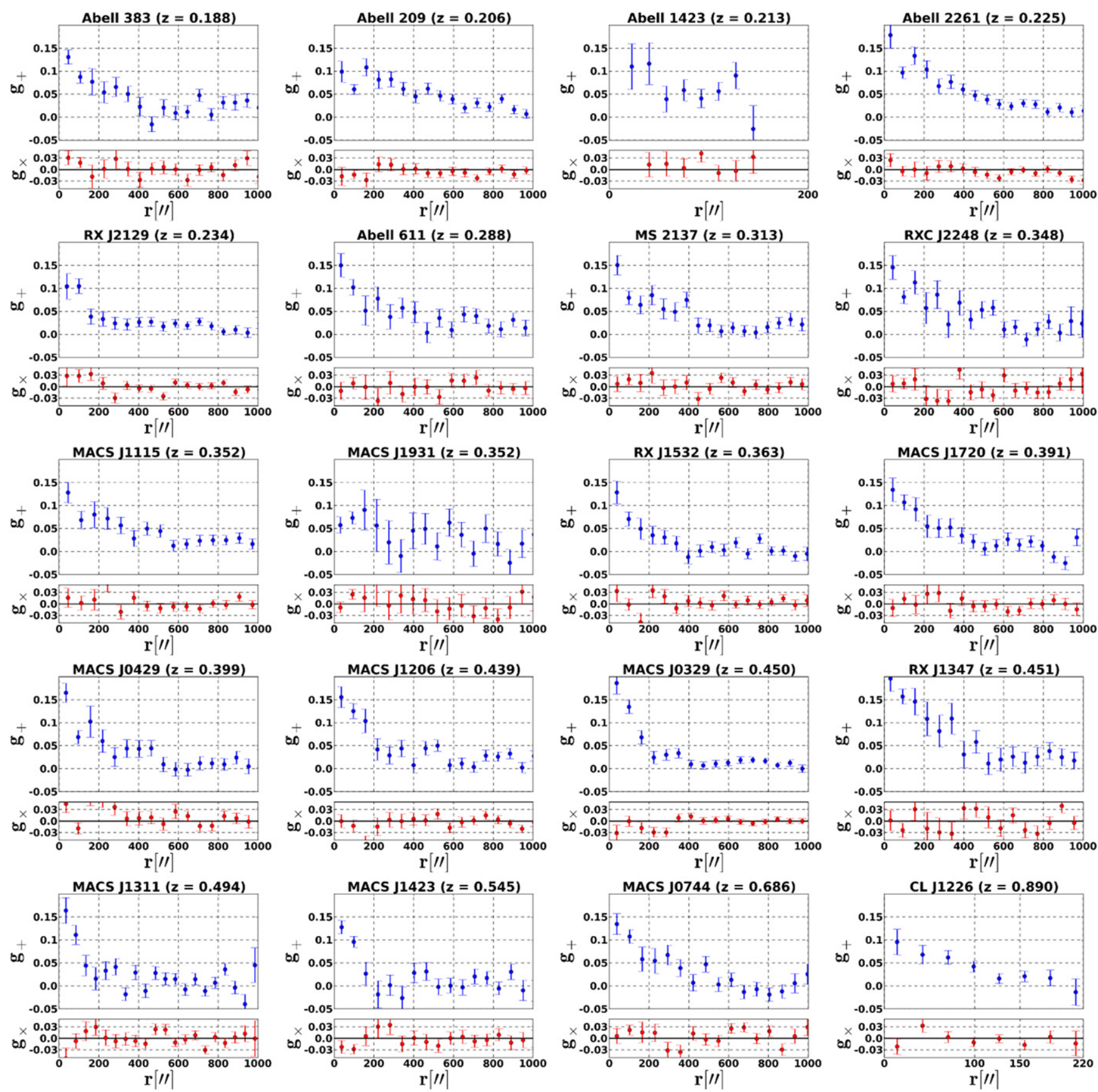

Figure 5. Shear profiles for the final ellipticity catalogs of 20 X-ray selected CLASH clusters. In the case of Abell 1423 and CL J1226 these catalogs derive from HST/ ACS images only. All other cases show combined HST/ACS and Subaru catalogs. The top plot of each panel shows the tangential shear profile, the bottom plot the cross shear profile with respect to the cluster center defined in Table $1.1 \sigma$ error bars were derived from 250 bootstrap resamplings of each input catalog.

pipeline has been used in Umetsu et al. (2012), Coe et al. (2012), Medezinski et al. (2013), and Umetsu et al. (2014).

We perform object detection and shape measurements using the IMCAT package developed by N. Kaiser based on the KSB (Kaiser et al. 1995) formalism. After initial object detection, close pairs are carefully identified and rejected to avoid the effects of object crowding on shape measurements (see Section 4.3 of Umetsu et al. 2014). The PSF anisotropy correction is performed according to the Umetsu et al. (2010) $\mathrm{KSB}+$ implementation using bright, un-saturated stars in the respective target fields. Following (Umetsu et al. 2010, see their Section 3.2.3) we calibrate KSB's isotropic correction factor as a function of object size and magnification by using galaxies detected with high significance $(\nu>30)$, in order to minimize the inherent shear calibration bias in the presence of noise. Finally, for each galaxy, shape measurements from different observation epochs and camera orientations are combined according to the prescription provided in Section 4.3 of Umetsu et al. (2014). The pipeline has been thoroughly tested with simulated Subaru/Suprime-Cam images (Massey et al. 2007; Oguri et al. 2012), where a multiplicative shear calibration bias of $|m| \simeq 5 \%$ and a residual shear offset of $c \sim 10^{-3}$ were found. We correct individual shear estimates for the residual multiplicative bias as $g \rightarrow g / 0.95$. 
Table 4

Ground-based Weak-lensing Constraints

\begin{tabular}{lcrrr}
\hline \hline Name & Shape-band & $N_{\text {gal }}$ & $\begin{array}{c}\rho_{\text {gal }} \\
\left(\operatorname{arcmin}^{-2}\right)\end{array}$ & $z_{\text {eff }}$ \\
\hline Abell 383 & & & 9.0 & 1.16 \\
Abell 209 & Ip & 7062 & 16.4 & 0.94 \\
Abell 1423 ${ }^{\mathrm{a}}$ & $\mathrm{Rc}$ & 14,694 & $\ldots$ & $\ldots$ \\
Abell 2261 & $\ldots$ & $\ldots$ & 18.8 & 0.89 \\
RX J2129+0005 & Rc & 15,429 & 24.5 & 1.16 \\
Abell 611 & Rc & 20,104 & 8.8 & 1.13 \\
MS 2137-2353 & Rc & 7872 & 11.6 & 1.23 \\
RXC J2248-4431 & Rc & 9133 & 5.5 & 1.05 \\
MACS J1115+0129 & WFI R & 4008 & 15.1 & 1.15 \\
MACS J1931-26 & Rc & 13,621 & 5.3 & 0.93 \\
RX J1532.8+3021 & Rc & 4343 & 16.6 & 1.15 \\
MACS J1720+3536 & Rc & 13,270 & 12.5 & 1.13 \\
MACS J0429-02 & Rc & 9855 & 12.0 & 1.25 \\
MACS J1206-08 & Rc & 9990 & 13.7 & 1.13 \\
MACS J0329-02 & Ic & 12,719 & 29.5 & 1.18 \\
RX J1347-1145 & Rc & 25,427 & 8.9 & 1.17 \\
MACS J1311-03 & Rc & 9385 & 20.2 & 1.07 \\
MACS J1423+24 & Rc & 13,748 & 9.8 & 0.98 \\
MACS J0744+39 & Rc & 7470 & 9.5 & 1.41 \\
CL J1226+3332 & Rc & 7561 & $\ldots$ & $\ldots$ \\
\hline & $\ldots$ & $\ldots$ & &
\end{tabular}

Note. These values derive from the comprehensive CLASH weak lensing work by Umetsu et al. (2014). Column explanations are identical to Table 3.

${ }^{a}$ No ground-based data of sufficient data quality in terms of seeing, exposure time and band coverage was available at the time this work was published.

After the catalog with shape measurements has been created, weak-lensing background sources for each cluster are selected following the description in Section 4.4 of Umetsu et al. (2014). Here we shortly summarize the process. The selection is based on the color-color (CC) technique by Medezinski et al. (2010), which uses empircal correlations in CC space, calibrated with evolutionary color tracks of galaxies (Medezinski et al. 2010; Hanami et al. 2012) and with the 30 band photo- $z$ distribution in the COSMOS field (Ilbert et al. 2009). This technique selects a pure sample of background galaxies with negligible contamination by foreground objects and cluster member galaxies. For the selection in CC space we usually use the Subaru/Suprime-Cam $B_{J} R_{C} z^{\prime}$ photometry and our conservative selection criteria usually yield about 12 galaxies $\operatorname{arcmin}^{-2}$.

\subsection{Combination of Shear Catalogs}

We combine the HST and ground-based catalogs into a single weak lensing catalog before the SaWLens reconstruction. In order to do so, we first correct for the different redshifts of the background populations in each catalog. We scale the two shear values in the $H S T$ catalogs with a factor

$$
\beta=\frac{D_{\mathrm{lS}} D_{H}}{D_{s} D_{\mathrm{lH}}},
$$

which accounts for the dependence of the shear on the lensing geometry. Here, $D_{1 \mathrm{~S}}\left(D_{1 \mathrm{H}}\right)$ is the angular diameter distance between the lens and the ground-based $(H S T)$ sample and $D_{S}$ $\left(D_{H}\right)$ is the angular diameter distance between the observer and the ground-based $(H S T)$ sample. After applying the correction $\beta$ to the HST shapes, we match the two catalogs by calculating the signal-to-noise weighted mean of sources which appear in both catalogs and by concatenating non-matching entries in the two catalogs. As a final cross-check we calculate the tangential $\left(g_{+}\right)$and cross-shear $\left(g_{\times}\right)$components in azimuthal bins around the cluster center, which we show in Figure 5.

\section{DENSITY PROFILES OF CLASH CLUSTERS}

Our mass reconstructions with associated error bars are used to fit NFW profiles to the surface-mass density distribution. Mass and concentration parameters for each of the X-ray selected CLASH clusters are the main result of our observational efforts.

\subsection{Final SaWLens Input and Results}

We summarize the basic parameters of each cluster reconstruction in Table 5, including input data, reconstructed field sizes and the refinement levels of the multi-scale grid. For two sample clusters, Abell 1423 and CL J1226, no multi-band wide-field weak lensing data with acceptable seeing and exposure time levels is available. In the case of CL J1226 this is less severe since we have access to a rather wide HST/ACS mosaic and, since the cluster resides at high redshift, the angular size of the reconstruction refers to a large physical size of the system. We therefore include CL J1226 in our following mass-concentration analysis, while we drop Abell 1423 from this sample.

The output of the reconstruction is the lensing potential on a multi-scale grid, which is then converted into a convergence or surface-mass density map via Equation (7). The convergence maps on a wide field for all sample clusters are shown in Figure 15. We base our follow-up analysis on these maps, together with a comprehensive assessment of their error budget.

\subsection{Error Estimation}

Non-parametric methods, especially when they include nonlinear constraints in the strong-lensing regime, do not offer a straight-forward way to analytically describe the error bars attached to reconstructed quantities (van Waerbeke 2000). We therefore follow the route of resampling the input catalogs to obtain error bars on our reconstructed convergence maps. The weak-lensing input is treated by bootstrap resampling the shear catalogs (see, e.g., Bradač et al. 2005a; Merten et al. 2011). For the strong-lensing input, we use two different criteria to resample our input catalogs. First, in each realization we radomly turn and off multiple images which were identified as only candidates by the Zitrin et al. (2009) method. The list of candidate system for each cluster has been published in Zitrin et al. (2014). Second, we randomly sample a redshift in the $95 \%$ confidence interval of the photo- $z$ estimate of each multiple-image system. Also these redshift intervals are provided in Zitrin et al. (2014). With this strategy of catalog re-sampling in the weak -and the strong-lensing regime, we sequentially repeat the reconstructions and create 1000 realizations for each cluster reconstruction. This number is chosen somewhat arbitrarily but is primarily driven by runtime considerations, due to the high numerical demands of nonparametric reconstruction methods. From the observed scatter in the ensemble of realizations we derive our error bars, e.g., in the form of a covariance matrix for binned convergence profiles, as we describe them in the following section. 
Table 5

Reconstruction Properties

\begin{tabular}{|c|c|c|c|c|c|c|c|}
\hline Name & Input Data ${ }^{a}$ & $\begin{array}{l}\text { Ground-based FOV } \\
\qquad\left(" x^{\prime \prime}\right)\end{array}$ & $\begin{array}{l}\text { HST FOV } \\
(" \times ")\end{array}$ & $\begin{array}{l}\Delta_{\text {ground }} \mathrm{b} \\
\left(^{\prime \prime}\right)\end{array}$ & $\begin{array}{c}\Delta_{\mathrm{ACS}}{ }^{\mathrm{c}} \\
\left({ }^{\prime \prime}\right)\end{array}$ & $\Delta_{\mathrm{SL}}{ }^{\mathrm{d}}$ & $\#_{\text {masks }}{ }^{\mathrm{e}}$ \\
\hline Abell 383 & $\mathrm{~S}, \mathrm{~A}, \mathrm{H}$ & $1500 \times 1500$ & $173 \times 173$ & 29 & 12 & 10 & 2 \\
\hline Abell 209 & $\mathrm{~S}, \mathrm{~A}, \mathrm{H}$ & $1500 \times 1500$ & $150 \times 150$ & 25 & 12 & 8 & 2 \\
\hline Abell 1423 & $\mathrm{H}$ & $\ldots$ & $200 \times 200$ & $\ldots$ & 13 & $\ldots$ & $\ldots$ \\
\hline Abell 2261 & $\mathrm{~S}, \mathrm{~A}, \mathrm{H}$ & $1500 \times 1500$ & $150 \times 150$ & 25 & 13 & 8 & 2 \\
\hline RX J2129+0005 & $\mathrm{S}, \mathrm{A}, \mathrm{H}$ & $1500 \times 1500$ & $150 \times 150$ & 25 & 10 & 8 & 3 \\
\hline Abell 611 & $\mathrm{~S}, \mathrm{~A}, \mathrm{H}$ & $1400 \times 1400$ & $168 \times 168$ & 28 & 10 & 9 & 1 \\
\hline MS 2137-2353 & $\mathrm{S}, \mathrm{A}, \mathrm{H}$ & $1500 \times 1500$ & $180 \times 180$ & 30 & 14 & 10 & 1 \\
\hline RXC J2248-4431 & $\mathrm{W}, \mathrm{A}, \mathrm{H}$ & $1500 \times 1500$ & $171 \times 171$ & 34 & 12 & 11 & 7 \\
\hline MACS J1115+0129 & $\mathrm{S}, \mathrm{A}, \mathrm{H}$ & $1500 \times 1500$ & $150 \times 150$ & 25 & 10 & 8 & 2 \\
\hline MACS J1931-26 & $\mathrm{S}, \mathrm{A}, \mathrm{H}$ & $1500 \times 1500$ & $179 \times 179$ & 36 & 10 & 10 & 0 \\
\hline RX J1532.8+3021 & $\mathrm{S}, \mathrm{A}$ & $1500 \times 1500$ & $155 \times 155$ & 26 & 10 & $\ldots$ & 3 \\
\hline MACS J1720+3536 & $\mathrm{S}, \mathrm{A}, \mathrm{H}$ & $1500 \times 1500$ & $150 \times 150$ & 25 & 9 & 8 & 3 \\
\hline MACS J0429-02 & $\mathrm{S}, \mathrm{A}, \mathrm{H}$ & $1500 \times 1500$ & $167 \times 167$ & 28 & 10 & 9 & 3 \\
\hline MACS J1206-08 & $\mathrm{S}, \mathrm{A}, \mathrm{H}$ & $1500 \times 1500$ & $150 \times 150$ & 25 & 12 & 8 & 0 \\
\hline MACS J0329-02 & $\mathrm{S}, \mathrm{A}, \mathrm{H}$ & $1500 \times 1500$ & $150 \times 150$ & 25 & 9 & 8 & 0 \\
\hline RX J1347-1145 & $\mathrm{S}, \mathrm{A}, \mathrm{H}$ & $1500 \times 1500$ & $180 \times 180$ & 30 & 12 & 10 & 1 \\
\hline MACS J1311-03 & $\mathrm{S}, \mathrm{A}, \mathrm{H}$ & $1500 \times 1500$ & $150 \times 150$ & 25 & 10 & 8 & 6 \\
\hline MACS J1423+24 & $\mathrm{S}, \mathrm{A}, \mathrm{H}$ & $1500 \times 1500$ & $155 \times 155$ & 26 & 8 & 8 & 2 \\
\hline MACS J0744+39 & $\mathrm{S}, \mathrm{A}, \mathrm{H}$ & $1500 \times 1500$ & $150 \times 150$ & 30 & 9 & 7 & 4 \\
\hline CL J1226+3332 & A, H & $\ldots$ & $300 \times 300$ & $\ldots$ & 8 & 6 & 0 \\
\hline
\end{tabular}

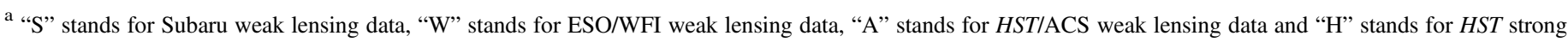
lensing data.

$\mathrm{b}$ The pixel size of the grid in the Subaru or ESO/WFI weak lensing regime.

c The pixel size of the grid in the HST/ACS weak lensing regime.

$\mathrm{d}$ The pixel size of the grid in the strong lensing regime.

e The number of masks in the reconstruction grid. There are necessary if bright stars blend large portions of the FOV.

\subsection{From Convergence Maps to NFW Profile Parameters}

Additional steps are needed to go from non-parametric maps of the surface-mass density distribution to an actual NFW fit of the halo. First, since we are interested in 1D density profiles, we apply an azimuthal binning scheme, with a bin pattern that follows the adaptive resolution of our multi-scale maps. The initial bin is limited by the resolution of the highest refinement level of the convergence grid (compare Table 5) and the outermost bin is set to a physical scale of $2 \mathrm{Mpc} / \mathrm{h}$ for each halo. We split the radial range defined by the two thresholds into 15 bins. An example for the cluster MACS J1720 is shown in Figure 6. An exception is CL J1226 with no available wide-field data from the ground, where we were limited to a maximum radius of $1.2 \mathrm{Mpc} / \mathrm{h}$ and where we divided the radial range into 11 bins. The center for the radial profile is the dominant peak in the convergence map. We applied this binning scheme to all 1000 convergence realizations for each cluster and derived the covariance matrix for the convergence bins. Both the convergence data points and the convergence matrix are shown in Figure 16.

To the convergence bins and the corresponding covariance matrix we fit a NFW profile given by Equation (1). We numerically project the NFW profile on a sphere along the lineof-sight and thereby introduce the assumption of spherical symmetry in our cluster mass profiles. This is certainly not justified for all sample clusters and may introduce biases. We discuss this issue in further detail in Section 6.

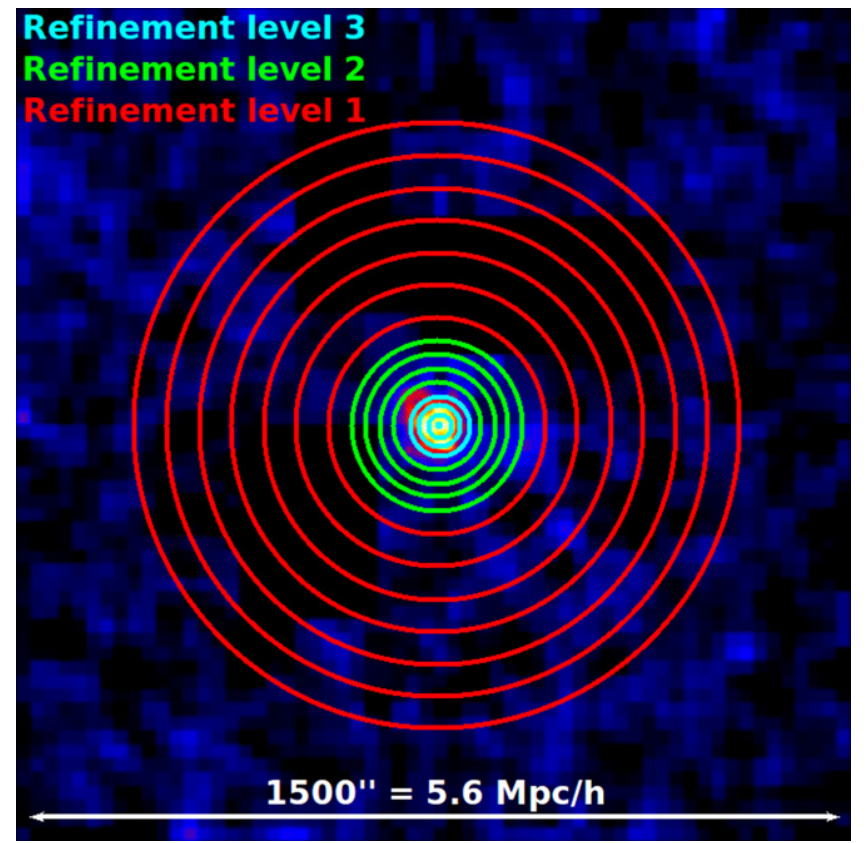

Figure 6. Adaptive binning scheme for the radial convergence profiles. Shown in this figure are the actual bins, overlaid on the cluster's convergence map, used to derive the convergence profile for the cluster MACS J1720 (compare Figure 16). The size of the bins follows the three levels of grid refinement as they are visualized in Figure 1 and listed in Table 5. 
Table 6

NFW Fits: General Parameters

\begin{tabular}{|c|c|c|c|c|c|c|}
\hline Name & $\begin{array}{c}\rho_{s} \pm \sqrt{\sigma_{\rho_{s} \rho_{s}}} \\
\left(10^{15} h^{2} M_{\odot} \mathrm{Mpc}^{-3}\right)\end{array}$ & $\begin{array}{c}r_{s} \pm \sqrt{\sigma_{r_{s} r_{s}}} \\
(\mathrm{Mpc} / \mathrm{h})\end{array}$ & $\begin{array}{c}\sigma_{\rho_{s} r_{s}} \\
\left(10^{15} h M_{\odot} \mathrm{Mpc}^{-2}\right)\end{array}$ & $\Delta_{\text {vir }}{ }^{\mathrm{a}}$ & $\begin{array}{c}r_{\mathrm{vir}} \\
(\mathrm{Mpc} / \mathrm{h})\end{array}$ & $\left(\chi^{2}\right)^{\mathrm{b}}$ \\
\hline Abell 383 & $2.47 \pm 0.59$ & $0.33 \pm 0.04$ & -0.02 & 111 & 1.86 & 2.0 \\
\hline Abell 209 & $1.14 \pm 0.29$ & $0.46 \pm 0.07$ & -0.02 & 112 & 1.95 & 2.9 \\
\hline Abell 1423 & $\ldots$ & $\ldots$ & $\ldots$ & 113 & $\ldots$ & $\ldots$ \\
\hline Abell 2261 & $1.07 \pm 0.41$ & $0.51 \pm 0.11$ & -0.05 & 114 & 2.26 & 3.7 \\
\hline RX J2129+0005 & $2.16 \pm 0.67$ & $0.30 \pm 0.05$ & -0.04 & 114 & 1.65 & 5.3 \\
\hline Abell 611 & $1.36 \pm 0.32$ & $0.41 \pm 0.06$ & -0.02 & 118 & 1.79 & 4.1 \\
\hline MS 2137-2353 & $1.14 \pm 0.20$ & $0.48 \pm 0.05$ & -0.01 & 120 & 1.89 & 1.5 \\
\hline RXC J2248-4431 & $1.24 \pm 0.34$ & $0.48 \pm 0.07$ & -0.02 & 122 & 1.92 & 1.3 \\
\hline MACS J1115+0129 & $0.61 \pm 0.17$ & $0.62 \pm 0.11$ & -0.02 & 123 & 1.78 & 5.6 \\
\hline MACS J1931-26 & $1.22 \pm 0.31$ & $0.41 \pm 0.07$ & -0.02 & 123 & 1.61 & 4.2 \\
\hline RX J1532.8+3021 & $1.16 \pm 0.52$ & $0.39 \pm 0.10$ & -0.05 & 123 & 1.47 & 6.9 \\
\hline MACS J1720+3536 & $2.44 \pm 0.84$ & $0.31 \pm 0.06$ & -0.05 & 125 & 1.61 & 4.2 \\
\hline MACS J0429-02 & $1.37 \pm 0.57$ & $0.41 \pm 0.08$ & -0.05 & 126 & 1.65 & 1.9 \\
\hline MACS J1206-08 & $2.60 \pm 0.94$ & $0.31 \pm 0.06$ & -0.05 & 128 & 1.63 & 4.9 \\
\hline MACS J0329-02 & $2.05 \pm 0.84$ & $0.33 \pm 0.08$ & -0.06 & 129 & 1.54 & 6.3 \\
\hline RX J1347-1145 & $2.10 \pm 0.90$ & $0.38 \pm 0.08$ & -0.07 & 129 & 1.80 & 3.2 \\
\hline MACS J1311-03 & $2.97 \pm 0.62$ & $0.24 \pm 0.03$ & -0.02 & 131 & 1.28 & 4.0 \\
\hline MACS J1423+24 & $3.70 \pm 1.83$ & $0.24 \pm 0.06$ & -0.11 & 134 & 1.34 & 6.4 \\
\hline MACS J0744+39 & $3.18 \pm 0.71$ & $0.28 \pm 0.04$ & -0.03 & 141 & 1.33 & 3.2 \\
\hline CL J1226+3332 & $3.72 \pm 0.83$ & $0.35 \pm 0.05$ & -0.04 & 150 & 1.57 & 2.7 \\
\hline
\end{tabular}

a The virial overdensity at cluster redshift in units of the critical density.

b The number of degrees of freedom is 10 in the case of CL J1226 and 14 for all other clusters.

Table 7

NFW Fits: Mass-concentration Parameters

\begin{tabular}{|c|c|c|c|c|c|c|c|c|}
\hline Name & $\begin{array}{c}M_{2500 c} \\
\left(10^{15} M_{\odot} / h\right)\end{array}$ & $c_{2500 c}$ & $\begin{array}{c}M_{500 c} \\
\left(10^{15} M_{\odot} / h\right)\end{array}$ & $c_{500 c}$ & $\begin{array}{c}M_{200 c} \\
\left(10^{15} M_{\odot} / h\right)\end{array}$ & $c_{200 c}$ & $\begin{array}{c}M_{\mathrm{vir}} \\
\left(10^{15} M_{\odot} / h\right)\end{array}$ & $c_{\mathrm{vir}}$ \\
\hline Abell 383 & $0.26 \pm 0.05$ & $1.3 \pm 0.3$ & $0.61 \pm 0.07$ & $2.9 \pm 0.7$ & $0.87 \pm 0.07$ & $4.4 \pm 1.0$ & $1.04 \pm 0.07$ & $5.6 \pm 1.3$ \\
\hline Abell 209 & $0.22 \pm 0.05$ & $0.9 \pm 0.3$ & $0.63 \pm 0.07$ & $2.1 \pm 0.6$ & $0.95 \pm 0.07$ & $3.3 \pm 0.9$ & $1.17 \pm 0.07$ & $4.3 \pm 1.1$ \\
\hline Abell 1423 & $\ldots$ & $\ldots$ & $\ldots$ & $\ldots$ & $\ldots$ & $\ldots$ & $\ldots$ & $\ldots$ \\
\hline Abell 2261 & $0.34 \pm 0.12$ & $0.9 \pm 0.4$ & $0.95 \pm 0.16$ & $2.2 \pm 0.9$ & $1.42 \pm 0.17$ & $3.4 \pm 1.4$ & $1.76 \pm 0.18$ & $4.4 \pm 1.8$ \\
\hline RX J2129+0005 & $0.18 \pm 0.03$ & $1.2 \pm 0.4$ & $0.43 \pm 0.04$ & $2.8 \pm 0.9$ & $0.61 \pm 0.06$ & $4.3 \pm 1.4$ & $0.73 \pm 0.07$ & $5.6 \pm 1.7$ \\
\hline Abell 611 & $0.21 \pm 0.04$ & $0.9 \pm 0.3$ & $0.57 \pm 0.04$ & $2.2 \pm 0.6$ & $0.85 \pm 0.05$ & $3.4 \pm 0.9$ & $1.03 \pm 0.07$ & $4.3 \pm 1.1$ \\
\hline MS 2137-2353 & $0.23 \pm 0.04$ & $0.8 \pm 0.2$ & $0.68 \pm 0.05$ & $2.0 \pm 0.4$ & $1.04 \pm 0.06$ & $3.1 \pm 0.6$ & $1.26 \pm 0.06$ & $4.0 \pm 0.7$ \\
\hline RXC J2248-4431 & $0.27 \pm 0.07$ & $0.8 \pm 0.3$ & $0.76 \pm 0.12$ & $2.0 \pm 0.6$ & $1.16 \pm 0.12$ & $3.2 \pm 0.9$ & $1.40 \pm 0.12$ & $4.0 \pm 1.1$ \\
\hline MACS J1115+0129 & $0.15 \pm 0.05$ & $0.5 \pm 0.2$ & $0.54 \pm 0.08$ & $1.4 \pm 0.4$ & $0.90 \pm 0.09$ & $2.3 \pm 0.7$ & $1.13 \pm 0.10$ & $2.9 \pm 0.9$ \\
\hline MACS J1931-26 & $0.16 \pm 0.03$ & $0.8 \pm 0.2$ & $0.45 \pm 0.04$ & $2.0 \pm 0.6$ & $0.69 \pm 0.05$ & $3.2 \pm 0.9$ & $0.83 \pm 0.06$ & $3.9 \pm 1.1$ \\
\hline RX J1532.8+3021 & $0.11 \pm 0.05$ & $0.8 \pm 0.4$ & $0.34 \pm 0.08$ & $1.9 \pm 0.9$ & $0.53 \pm 0.08$ & $3.0 \pm 1.4$ & $0.64 \pm 0.09$ & $3.8 \pm 1.7$ \\
\hline MACS J1720+3536 & $0.22 \pm 0.06$ & $1.2 \pm 0.5$ & $0.53 \pm 0.08$ & $2.8 \pm 1.0$ & $0.75 \pm 0.08$ & $4.3 \pm 1.4$ & $0.88 \pm 0.08$ & $5.2 \pm 1.7$ \\
\hline MACS J0429-02 & $0.19 \pm 0.11$ & $0.9 \pm 0.4$ & $0.53 \pm 0.13$ & $2.1 \pm 0.9$ & $0.80 \pm 0.14$ & $3.3 \pm 1.3$ & $0.96 \pm 0.14$ & $4.0 \pm 1.6$ \\
\hline MACS J1206-08 & $0.25 \pm 0.08$ & $1.2 \pm 0.5$ & $0.60 \pm 0.11$ & $2.8 \pm 1.0$ & $0.86 \pm 0.11$ & $4.3 \pm 1.5$ & $1.00 \pm 0.11$ & $5.2 \pm 1.7$ \\
\hline MACS J0329-02 & $0.20 \pm 0.06$ & $1.1 \pm 0.4$ & $0.50 \pm 0.09$ & $2.5 \pm 1.1$ & $0.73 \pm 0.10$ & $3.8 \pm 1.6$ & $0.86 \pm 0.11$ & $4.7 \pm 1.9$ \\
\hline RX J1347-1145 & $0.31 \pm 0.13$ & $1.1 \pm 0.5$ & $0.79 \pm 0.19$ & $2.5 \pm 1.1$ & $1.16 \pm 0.19$ & $3.9 \pm 1.5$ & $1.35 \pm 0.19$ & $4.7 \pm 1.8$ \\
\hline MACS J1311-03 & $0.14 \pm 0.02$ & $1.3 \pm 0.3$ & $0.32 \pm 0.19$ & $2.9 \pm 0.6$ & $0.46 \pm 0.03$ & $4.4 \pm 1.0$ & $0.53 \pm 0.04$ & $5.3 \pm 1.1$ \\
\hline MACS J1423+24 & $0.18 \pm 0.08$ & $1.4 \pm 0.8$ & $0.41 \pm 0.06$ & $3.1 \pm 0.8$ & $0.57 \pm 0.10$ & $4.7 \pm 1.2$ & $0.65 \pm 0.11$ & $5.7 \pm 2.8$ \\
\hline MACS J0744+39 & $0.20 \pm 0.03$ & $1.2 \pm 0.3$ & $0.49 \pm 0.04$ & $2.7 \pm 0.6$ & $0.70 \pm 0.04$ & $4.1 \pm 1.0$ & $0.79 \pm 0.04$ & $4.8 \pm 1.1$ \\
\hline CL J1226+3332 & $0.43 \pm 0.07$ & $1.1 \pm 0.3$ & $1.08 \pm 0.09$ & $2.6 \pm 0.6$ & $1.56 \pm 0.10$ & $4.0 \pm 0.9$ & $1.72 \pm 0.11$ & $4.5 \pm 1.1$ \\
\hline
\end{tabular}

We perform the profile fitting using the least-squares formalism by minimizing

$$
\chi^{2}(\boldsymbol{p})=\sum_{i, j=0}^{N_{\mathrm{bin}}}\left(\kappa_{\mathrm{bin}}-\kappa(\boldsymbol{p})\right)_{i} \mathcal{C}_{i j}^{-1}\left(\kappa_{\mathrm{bin}}-\kappa(\boldsymbol{p})\right)_{j},
$$

where $\boldsymbol{p}=\left(\rho_{s}, r_{s}\right)$ and $\mathcal{C}$ is the covariance matrix of the binned data. The numerical fitting is performed using the open-source library levmar ${ }^{35}$ and by making use of the Cholesky decomposition of $\mathcal{C}^{-1}$. The best-fit parameters, the corresponding covariance matrix and the fitting norm is reported in Table 6. We use these values, together with Equations (2) and (3) to find our final mass and concentration values at several different radii. We report this central result of our work in

\footnotetext{
35 http://users.ics.forth.gr/lourakis/levmar/
} 


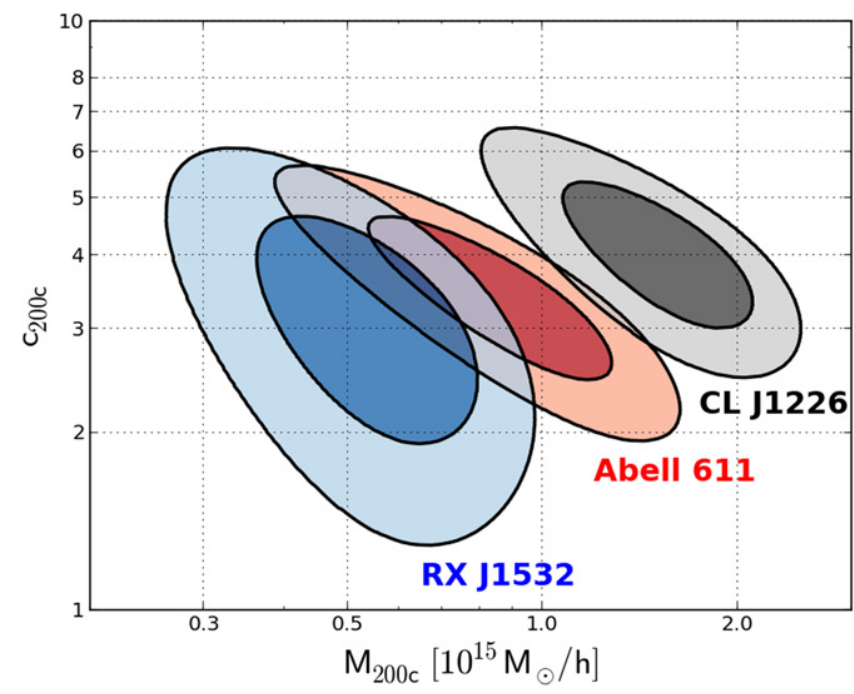

Figure 7. Likelihood of NFW fits in the c-M plane. The cluster Abell 611 represents a typical CLASH cluster at an intermediate redshift with the full set of lensing constraints available. RX J1532 is the only cluster in this c-M analysis without strong lensing constraints and CL J1226 is the only cluster in the sample without available Subaru weak lensing data. The inner and outer contours show the $68 \%$ and $95 \%$ confidence levels.

Table 7. To visualize degeneracies and to show the information gain when including strong-lensing features into the reconstruction we explore the likelihood in the c-M plane for three CLASH clusters in Figure 7.

\subsection{Sources of Systematic Error}

Before moving on in our analysis we want to discuss possible sources of systematic error. In the strong-lensing regime there is the possibility of false identification of multipleimage systems. In the case of CLASH, many strong-lensing features have no spectroscopic confirmation. However, CLASH can rely on 16-band $H S T$ photometry for photo- $z$ determination. Finally, those systems which are only identified as candidates by the Zitrin et al. (2009) method for image identification are considered as such in our extensive bootstrap approach. Another problem for strong lensing is the shift of multiple-image positions by contributions of projected large scale structure. This has been pointed out recently in D'Aloisio \& Natarajan (2011), Host (2012). However, as it was shown by the latter authors, the expected shift in image postion is well below our minimum reconstruction pixel scale of $5^{\prime \prime}-10^{\prime \prime}$ for the different clusters (compare Figure 2).

We address shape scatter in the weak-lensing catalogs with the adaptive-averaging approach of the SawLens method and by bootstrapping the weak-lensing input. Foreground contamination of the shear catalogs is another serious concern which will lead to a significant dilution of the weak lensing signal. In the HST images this is controlled by reliable photometric redshifts. However, there is the possibility of remaining contamination by cluster members in the crowded fields and by stars falsely identified as galaxies. Background selection in the ground-based catalogs is more difficult due to the smaller number of photometric bands. Hence, we use the Medezinski et al. (2010) method of background selection in color-color space which was optimized to avoid weak lensing dilution.
The aforementioned mass-sheet degeneracy (Equation (11)) is another concern for systematic error. We described the way of breaking this degeneracy in this work and tested the validity of this approach against numerical simulations (Merten et al. 2009; Meneghetti et al. 2010b). However, these simulations represent a much smaller sample and derive from a different selection function than the CLASH sample. Furthermore, the box-sizes of these cluster re-simulations is limited and therefore these tests do not guarantee that our treatment of the mass-sheet degeneracy produces fully unbiased mass estimates.

We have not commented yet on the dominant density peak in the lensing reconstruction as our center choice for the radial density profile. Because of the inclusion of strong lensing constraints, this peak position has an uncertainty of only a few arcsec (e.g., Bradač et al. 2006; Merten et al. 2011), but one might argue that e.g., the position of the cluster's BCG is a more accurate tracer of the potential minimum. However, our pixel resolution is of the order of $\sim 5^{\prime \prime}$ and $\mathrm{BCG}$ position and the peak in the surface-mass density coincide or are offset by one or two pixels.

More important is the effect of uncorrelated large scale structure (e.g., Hoekstra et al. 2011, and references therein) and tri-axial halo shape (Becker \& Kravtsov 2011) which is picked up by our lensing reconstruction. Becker \& Kravtsov (2011) claim that these effects introduce only small biases in the mass determination but increase the scatter by up to $20 \%$ with triaxial shape being the dominant component. We do not seek to correct for these effects directly but adapt our way of analyzing numerical simulations accordingly (Section 6). In order to quantify our total error budget, we refer to Meneghetti et al. (2010b) where our SaWLens approach of mass-modeling underwent a thorough testing procedure in a controlled, simulated environment. Based on these results we report a systematic error between 5\%-10\%, depending on the level of substructure in the halo of interest.

\subsection{Comparison to Other Analyses}

As a final consistency check we look into 15 clusters that we have in common with the Weighing the Giants (dubbed as WtG hereafter) project (Applegate et al. 2014; Kelly et al. 2014; von der Linden et al. 2014) and the 16 clusters we have in common with the CLASH shear and magnification study by Umetsu et al. (2014; dubbed as U14 hereafer). For the direct comparison to $\mathrm{WtG}$ and $\mathrm{U} 14$ we calculate the enclosed mass within $1.5 \mathrm{Mpc}$ of the cluster center following Applegate et al. (2014). This is to have a meaningful comparison at a fixed phyiscal radius and not to have to correct for the different mass apertures. We also used the cosmology of WtG to derive the masses for this comparison and show the mass comparison for the three data sets in Figure 8. We find median values for the ratios $M_{\text {SaWLens }} / M_{\mathrm{WtG}}$ and $M_{\mathrm{WtG}} / M_{\text {SaWLens }}$ of $0.88_{-0.06}^{+0.10}$ and $1.12_{-0.10}^{+0.06}$, respectively. The upper and lower bounds derive from the third and first quartile of the ratio sample. For the unweighted geometric mean ${ }^{36}$ of these ratios we find $0.94 \pm 0.11$. The respective numbers for the 16 cluster comparison to U14 are $0.93_{-0.09}^{+0.14}$ and $1.08_{-0.14}^{+0.11}$ for the median of the ratios $M_{\text {SaWLens }} / M_{\mathrm{U} 14}$ and $M_{\mathrm{U} 14} / M_{\text {SaWLens. }}$ The geometric mean of the ratios yields $0.95 \pm 0.06$. Although we see

\footnotetext{
${ }^{36}$ The geometric mean satisfies $\langle X / Y\rangle=1 /\langle Y / X\rangle$ for the ratio of samples $X$ and $Y$.
} 


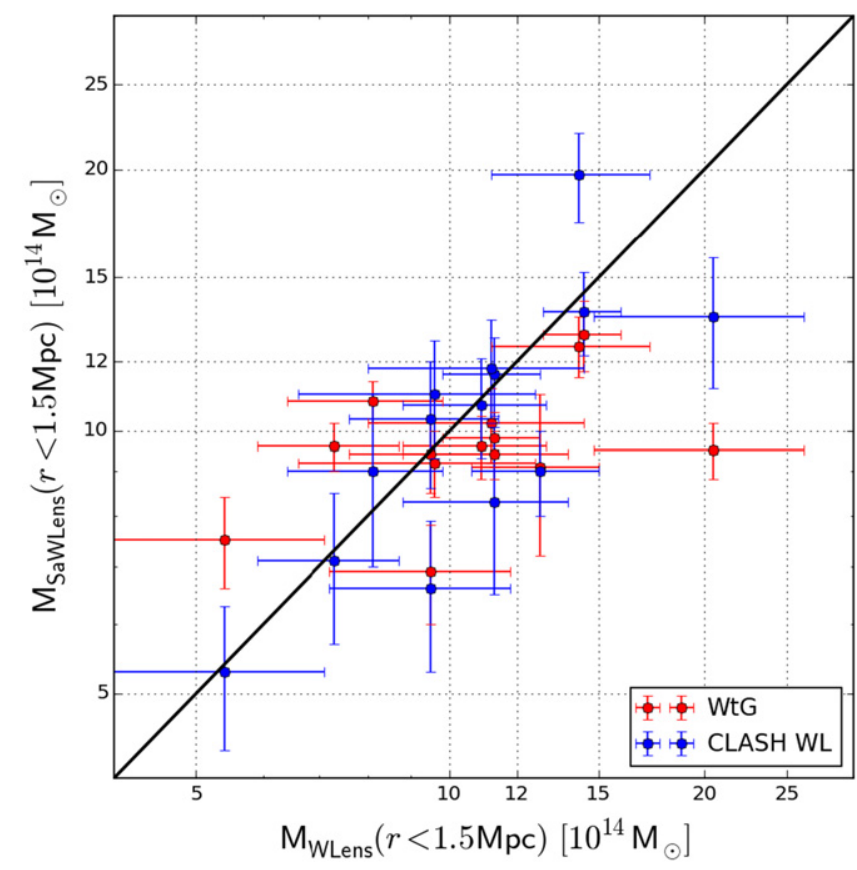

Figure 8. Comparison between our analysis and other weak-lensing studies. The red data points show clusters in common with $\mathrm{WtG}$ and the blue data points show the overlap with Umetsu et al. (2014). On the y-axis we plot enclosed SaWLens masses within a radius of $1.5 \mathrm{Mpc}$ from the cluster center. The $x$-axis shows equivalent masses from $\mathrm{WtG}$ and $\mathrm{U} 14$, respectively. The black line indicates a one-to-one agreement.

significant scatter between the different studies, there is general agreement but we have to point out that our analysis and U14 use identical Subaru weak-lensing catalogs. In the following subsections we report these different mass estimates cluster by cluster and also consult other studies of a specific object.

For a comparison of our mass estimates to X-ray masses we want to refer to the recent work by Donahue et al. (2014), where X-ray mass profiles from Chandra and XMM-Newton were compared to the lensing-derived profiles of U14 and to our profiles reported in Table 6. Donahue et al. (2014) find that Chandra masses at $0.5 \mathrm{Mpc}$, assuming hydrostatic equilibrium, are on average $11 \%$ larger than the masses presented in this work for a sample of 10 clusters that the studies have in common. For hydrostatic masses from XMM-Newton at $0.5 \mathrm{Mpc}$, the opposite trend was found, where for a sample of 13 common clusters our lensing masses were $18 \%$ higher than the X-ray masses.

\subsubsection{Abell 383}

This cluster at $z=0.188$ is one of the first clusters studied by CLASH Zitrin et al. (2011). In the mass comparison with $\mathrm{WtG}$, our value of $M_{1.5 \mathrm{Mpc}}=9.6 \pm 0.6 \times 10^{14} M_{\odot}$ is larger than $M_{1.5 \mathrm{Mpc}}=7.3 \pm 1.4 \times 10^{14} M_{\odot}$ of $\mathrm{WtG}$ at the $\sim 1.5 \sigma$ level, which is consistent with the findings of U14 with $M_{1.5 \mathrm{Mpc}}=7.1 \pm 1.4 \times 10^{14} M_{\odot}$. To have another independent study we refer to Newman et al. (2013) who find $M_{1.7 \mathrm{Mpc}}=6.6_{-1.1}^{+1.5} \times 10^{14} M_{\odot}$ for this object. The mass from our model at the same radius yields $M_{1.7 \mathrm{Mpc}}=$ $10.7 \pm 0.7 \times 10^{14} M_{\odot}$, which is again in some tension. The reason for this discrepancy is unclear, especially since Abell 383 is thought to be a rather relaxed object. However, the tension is also not very significant.

\subsubsection{Abell 209}

Our lensing reconstruction of this system at $z=0.206$ suggests a rather massive but regular system with respect to the morphology in its surface-mass density map. This is supported by our derived mass of $M_{1.5 \mathrm{Mpc}}=9.8 \pm$ $0.7 \times 10^{14} M_{\odot}$, which compares well to the findings of U14 with $M_{1.5 \mathrm{Mpc}}=11.6 \pm 1.2 \times 10^{14} M_{\odot}$ and $\mathrm{WtG}$ with $M_{1.5 \mathrm{Mpc}}=$ $11.3 \pm 1.5 \times 10^{14} M_{\odot}$. An earlier study by Paulin-Henriksson et al. (2007) reports $M_{1.8 \mathrm{Mpc}}=7.7_{-2.7}^{+4.3} \times 10^{14} M_{\odot}$ and we compare to our result at the same radius and using the same cosmology of $M_{1.8 \mathrm{Mpc}}=11.7 \pm 0.9 \times 10^{14} M_{\odot}$, which shows no significant tension but a higher mass. We would expect such a result since the background selection of weak-lensing galaxies in Paulin-Henriksson et al. (2007) was based on single-band data which is plagued by severe dilution effects (Medezinski et al. 2007, 2008).

\subsubsection{Abell 2261}

Abell 2261 at $z=0.225$ is one of the most massive clusters in our sample with one of the largest BCGs observed (Postman et al. 2012b). Our mass estimate of $M_{1.5 \mathrm{Mpc}}=12.9 \pm 1.2 \times 10^{14} M_{\odot}$ is in excellent agreement with $M_{1.5 \mathrm{Mpc}}=13.7 \pm 1.5 \times 10^{14} M_{\odot}$ by U14 and $M_{1.5 \mathrm{Mpc}}=14.4 \pm$ $1.5 \times 10^{14} M_{\odot}$ by WtG. An earlier CLASH study by Coe et al. (2012) derived a virial mass of $M_{\mathrm{vir}}=22.1_{2.3}^{+2.5} \times 10^{14} M_{\odot}$, which compares well to our virial mass estimate of $M_{\mathrm{vir}}=25.1 \pm 2.5 \times 10^{14} M_{\odot}$.

\subsubsection{RXJ 2129}

This low-mass system at $z=0.234$ shows some interesting morphology in the surface-mass density map of its core (see Figure 15). Since our fitting range starts at smaller radii, this might explain why our mass of $M_{1.5 \mathrm{Mpc}}=7.5 \pm 0.9 \times 10^{14} M_{\odot}$ is larger, although insignificantly, than $M_{1.5 \mathrm{Mpc}}=5.4 \pm$ $1.7 \times 10^{14} M_{\odot}$ by $\mathrm{WtG}$ and shows some more tension with $M_{1.5 \mathrm{Mpc}}=5.3 \pm 1.0 \times 10^{14} M_{\odot}$ by $\mathrm{U} 14$.

\subsubsection{Abell 611}

Also Abell 611 at $z=0.288$ was studied by Newman et al. (2013) where a mass of $M_{1.76 \mathrm{Mpc}}=8.3_{-1.2}^{+1.5} \times 10^{14} M_{\odot}$ is reported. At this radius we find $M_{1.76 \mathrm{Mpc}}=10.9 \pm$ $1.1 \times 10^{14} M_{\odot}$, in good agreement with this former study, and also our value of $M_{1.5 \mathrm{Mpc}}=9.4 \pm 0.9 \times 10^{14} M_{\odot}$ is in agreement with $M_{1.5 \mathrm{Mpc}}=9.5 \pm 1.9 \times 10^{14} M_{\odot}$ by WtG. This picture is further confirmed by $\mathrm{U} 14$ with $M_{1.5 \mathrm{Mpc}}=$ $10.3 \pm 1.7 \times 10^{14} M_{\odot}$.

\subsubsection{MS 2137}

MS 2137 is a well-studied cluster at $z=0.313$ for which we find a rather high mass of $M_{1.5 \mathrm{Mpc}}=10.8 \pm 0.6 \times 10^{14} M_{\odot}$, compared to $M_{1.5 \mathrm{Mpc}}=8.1 \pm 1.7 \times 10^{14} M_{\odot}$ by $\mathrm{WtG}$ and $M_{1.5 \mathrm{Mpc}}=9.0 \pm 2.0 \times 10^{14} M_{\odot}$ by U14. Also Newman et al. (2013) looked at this system and found $M_{1.32 \mathrm{Mpc}}=$ $3.6_{-0.8}^{+1.3} \times 10^{14} M_{\odot}$. For this aperture however, we find 
$M_{1.32 \mathrm{Mpc}}=6.4 \pm 0.4 \times 10^{14} M_{\odot}$, indicating a significantly higher mass from our reconstruction.

\subsection{7. $R X C J 2248$}

This clusters is another very massive system at $z=0.348$ and part of the HST Frontier Fields initiative. ${ }^{37}$ RXCJ 2248 is not part of the $\mathrm{WtG}$ program but our mass of $M_{1.5 \mathrm{Mpc}}=11.8 \pm 0.7 \times 10^{14} M_{\odot}$ agrees well with U14's $M_{1.5 \mathrm{Mpc}}=12.0 \pm 2.0 \times 10^{14} M_{\odot}$ and as an independent cross-check we refer to Melchior et al. (2015) presenting a cluster study of the Science Verification Data of the Dark Energy Survey (The Dark Energy Survey Collaboration 2005). Although they do not provide the exact radius of their mass-measurement aperture, they report $M_{200 c}=17.6 \pm$ $4.5 \times 10^{14} M_{\odot}$, which is in good agreement with our value of $M_{200 c}=16.6 \pm 1.7 \times 10^{14} M_{\odot}$, when assuming that the enclosed-mass apertures are similar. With the same assumption we finally quote another recent mass estimate by Gruen et al. (2013), which yields $M_{200 c}=22.8_{-4.7}^{+6.6} \times 10^{14} M_{\odot}$, also in agreement with the other mass estimates, although it has to be noted that the Gruen et al. (2013) result is based on the same imaging data as our work.

\subsubsection{MACS $J 1115$}

For this system at $z=0.352$ we also find excellent agreement between all mass measurements. Our mass of $M_{1.5 \mathrm{Mpc}}=9.6 \pm 0.8 \times 10^{14} M_{\odot}$ compares to $M_{1.5 \mathrm{Mpc}}=10.9 \pm$ $2.1 \times 10^{14} M_{\odot}$ by $\mathrm{WtG}$ and $M_{1.5 \mathrm{Mpc}}=10.7 \pm 1.4 \times 10^{14} M_{\odot}$ by U14.

\subsubsection{MACS J1931}

For this cluster at $z=0.352$ we have no overlap with the $\mathrm{WtG}$ progam but $\mathrm{U} 14$ find $M_{1.5 \mathrm{Mpc}}=11.0 \pm 2.9 \times 10^{14} M_{\odot}$, which is consistent with our value $M_{1.5 \mathrm{Mpc}}=8.3 \pm$ $1.0 \times 10^{14} M_{\odot}$ within the large uncertainties. This is due to a low number of useable background galaxies in the MACS J1931 field at a low Galactic latitude (see Section 4.4 of Umetsu et al. 2014).

\subsubsection{0. $R X J 1532$}

Situated at $z=0.363$, this is another low-mass system for which we find $M_{1.5 \mathrm{Mpc}}=6.9 \pm 0.9 \times 10^{14} M_{\odot}$, in good agreement with U14 who quote $M_{1.5 \mathrm{Mpc}}=6.6 \pm 1.3 \times 10^{14} M_{\odot}$ and consistent with the $\mathrm{WtG}$ value $M_{1.5 \mathrm{Mpc}}=9.5 \pm 2.3 \times 10^{14} M_{\odot}$.

\subsubsection{MACS $J 1720$}

MACS J1720 at $z=0.391$ shows good agreement between the mass from $\mathrm{WtG}$ with $M_{1.5 \mathrm{Mpc}}=9.6 \pm 3.0 \times 10^{14} M_{\odot}$, U14 with $M_{1.5 \mathrm{Mpc}}=11.0 \pm 1.7 \times 10^{14} M_{\odot}$ and our value of $M_{1.5 \mathrm{Mpc}}=9.2 \pm 0.8 \times 10^{14} M_{\odot}$.

\subsubsection{MACS J0429}

For MACS J0429 at $z=0.399$ we find a higher mass of $M_{1.5 \mathrm{Mpc}}=9.4 \pm 0.6 \times 10^{14} M_{\odot}$ than U14 with $M_{1.5 \mathrm{Mpc}}=8.3 \pm$

\footnotetext{
${ }^{37}$ http://www.stsci.edu/hst/campaigns/frontier-fields/
}

$1.8 \times 10^{14} M_{\odot}$, but a lower mass than WtG with $M_{1.5 \mathrm{Mpc}}=$ $11.3 \pm 2.5 \times 10^{14} M_{\odot}$. All quoted values are in agreement within their $68 \%$ confidence interval error bars.

\subsubsection{MACS J1206}

The well-studied CLASH cluster MACS J1206 at $z=0.439$ was the first cluster analyzed with our new CLASH Subaru weak-lensing pipeline in Umetsu et al. (2012). In this earlier work we report a virial mass of $M_{\text {vir }}=16.4_{-4.0}^{+4.9} \times 10^{14} M_{\odot}$, which is in good agreement with our vitial mass $M_{\text {vir }}=14.3 \pm 1.6 \times 10^{14} M_{\odot}$. The same is true when comparing to the analysis of Biviano et al. (2013) which is based on the dynamics of cluster member galaxies and yields $M_{2.0 \mathrm{Mpc}}=14.0 \pm 2.0 \times 10^{14} M_{\odot}$ in excellent agreement with our value of $M_{2.0 \mathrm{Mpc}}=12.9 \pm 1.3 \times 10^{14} M_{\odot}$, after we adopt the background cosmology of Biviano et al. (2013). When comparing to the more recent analysis of $\mathrm{U} 14$ and $\mathrm{WtG}$ at smaller radius, we find $M_{1.5 \mathrm{Mpc}}=10.2 \pm 1.0 \times 10^{14} M_{\odot}$ for our work, $M_{1.5 \mathrm{Mpc}}=11.8 \pm 1.6 \times 10^{14} M_{\odot}$ for U14 and $M_{1.5 \mathrm{Mpc}}=11.2 \pm 3.2 \times 10^{14} M_{\odot}$ for $\mathrm{WtG}$, respectively.

\subsubsection{MACS J0329}

This cluster at $z=0.450$ shows quite some interesting morphology in its central surface-mass density map, with a very broad and flat inner core, which was already reported in Zitrin et al. (2012a). This might explain the somewhat lower mass from our NFW fit which yields $M_{1.5 \mathrm{Mpc}}=9.1 \pm$ $1.9 \times 10^{14} M_{\odot}$ compared to $M_{1.5 \mathrm{Mpc}}=12.8 \pm 2.2 \times 10^{14} M_{\odot}$ from WtG. However, U14 find $M_{1.5 \mathrm{Mpc}}=9.1 \pm 1.0 \times 10^{14} M_{\odot}$, which is in excellent agreement with our result.

\subsubsection{RXJ 1347}

This well-known cluster at $z=0.451$ was subject to many strong-lensing studies (e.g., Bradač et al. 2005a; Halkola et al. 2008, and references therein) at small radii. In order to compare our estimate for the total mass, for which we find $M_{1.5 \mathrm{Mpc}}=12.5 \pm 1.0 \times 10^{14} M_{\odot}$, we quote the number by $\mathrm{WtG} \quad M_{1.5 \mathrm{Mpc}}=14.2 \pm 3.0 \times 10^{14} M_{\odot}$, which is in good agreement. However, U14 find a much larger value of $M_{1.5 \mathrm{Mpc}}=19.7 \pm 2.3 \times 10^{14} M_{\odot}$. In an ealier study, Broadhurst et al. (2008) quote a virial mass of $M_{\text {vir }}=14.7_{-2.3}^{+2.6} \times$ $10^{14} M_{\odot}$, which is interestingly enough smaller than our estimate of $M_{\mathrm{vir}}=19.3 \pm 2.7 \times 10^{14} M_{\odot}$. The reason for this inconsistently diverting mass estimates is not entirely clear to us, but we suspect that the different fitting ranges for the NFW fits might play a crucial role in the mass estimates for disturbed systems such as RX J1347.

\subsubsection{MACS J1423}

For this system at $z=0.545$ we only have the comparison to the $\mathrm{WtG}$ analysis. Depending on the method of weak-lensing background selection, they report quite different mass estimates in Applegate et al. (2014). For a color-selected background selection they find $M_{1.5 \mathrm{Mpc}}=3.7 \pm 2.8 \times 10^{14} M_{\odot}$, while for a selection based on the full photo- $z$ distribution function they quote $M_{1.5 \mathrm{Mpc}}=8.8 \pm 3.6 \times 10^{14} M_{\odot}$. Our value of $M_{1.5 \mathrm{Mpc}}=$ 
$7.9 \pm 1.2 \times 10^{14} M_{\odot}$, agrees well with the photo- $z$ selected mass by $\mathrm{WtG}$.

\subsubsection{MACS J0744}

This cluster at a fairly high redshift of $z=0.686$ shows very different mass estimates, which might again be related to the extreme dynamical state this cluster is in. Korngut et al. (2011) report a very disturbed morphology for the matter distribution in the core based on high-resolution SZ and X-ray observations. We find a mass of $M_{1.5 \mathrm{Mpc}}=9.5 \pm 0.7 \times 10^{14} M_{\odot}$, which is significantly lower than $M_{1.5 \mathrm{Mpc}}=20.5 \pm 5.7 \times$ $10^{14} M_{\odot}$ by $\mathrm{WtG}$ and $M_{1.5 \mathrm{Mpc}}=13.5 \pm 2.3 \times 10^{14} M_{\odot}$ by U14. A recent study by Sereno et al. (2015) finds $M_{200 c}=13.6 \pm 3.3 \times 10^{14} M_{\odot}$ in marginal agreement with our finding of $M_{200 c}=10.0 \pm 0.6 \times 10^{14} M_{\odot}$. Although the Sereno et al. (2015) analysis differs in the way the NFW fit was performed, it is not independent of our analysis since it uses the radial shear profile reported in U14 and an estimate on the critical curve of the system from our Table 2. The largely different mass estimates for this interesting cluster render it as ideal target for further multi-wavelength studies.

\subsubsection{CLJ 1226}

For the highest-redshift system in our sample at $z=0.89$ we compare to the study by Jee \& Tyson (2009) who report a total mass of the system of $M_{1.12 \mathrm{Mpc}}=14.0 \pm 2.0 \times 10^{14} M_{\odot}$. Using their cosmological background model and deriving the enclosed mass for the same aperture radius we find $M_{1.12 \mathrm{Mpc}}=18.9 \pm 1.5 \times 10^{14} M_{\odot}$. This $35 \%$ larger mass is in mild tension with the previous study. However, as stated in Jee \& Tyson (2009) and confirmed by Korngut et al. (2011), also this system seems to be in a disturbed, merging state. As we have seen for the examples of RX J1347 and MACS J0744, such systems are prone to differing mass estimates, especially if different fitting ranges were used, which renders also this system as an interesting candidate for additional, independent studies.

\section{GENERAL C-M ANALYSIS}

We now derive a c-M relation from our 19 X-ray selected CLASH clusters and compare the observed values to the theoretical expectations from the literature. In the following, we will quote mass and concentration values which refer to a halo radius of $r_{200 c}$.

\subsection{The c-M Relation from CLASH}

In Figure 9 we visualize the CLASH data points from Table 7 in the $\mathrm{c}-\mathrm{M}$ plane. A general statistical summary of the data is shown in Table 10. In order to derive a c-M relation, we choose a parametrization following Duffy et al. (2008), but with pivot mass and redshift matched to our sample,

$$
\begin{aligned}
c_{200 c}\left(M_{200 c}, z\right) & =A \times\left(\frac{1.37}{1+z}\right)^{B} \\
& \times\left(\frac{M_{200 c}}{8 \times 10^{14} M_{\odot} / h}\right)^{C} .
\end{aligned}
$$
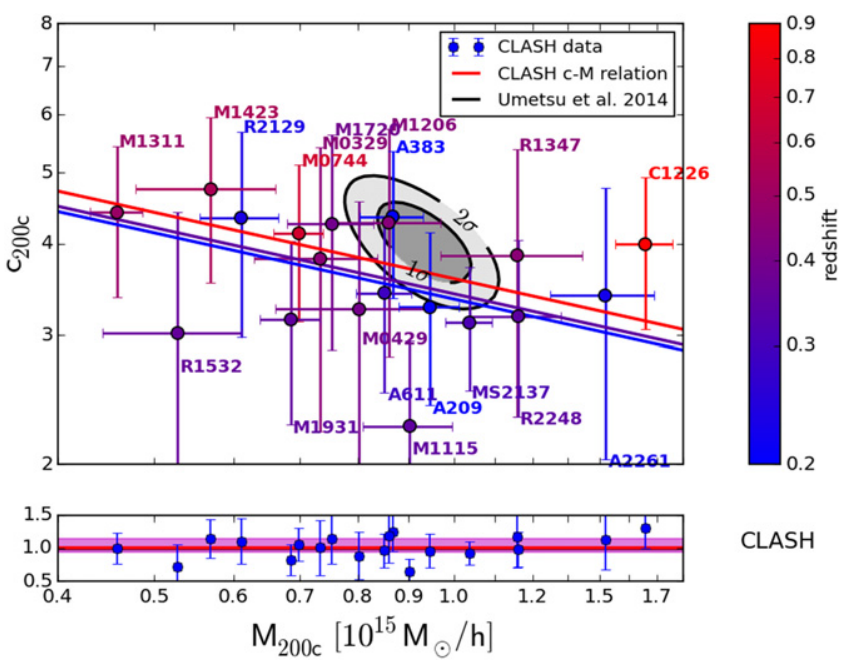

CLASH

Figure 9. Concentrations and masses from CLASH. The labeled data points in the top panel show each CLASH cluster in the $M_{200 c}-c_{200 c}$ plane. The solid lines show the best-fit c-M relation to the CLASH data for $z=0.2$ (blue), $z=0.35$ (purple) and $z=0.9$ (red). The color of data points and lines encodes the redshift of the CLASH clusters or the c-M relation. Overplotted with the gray contours is the concentration-mass analysis of Umetsu et al. (2014) for a redshift of $z \simeq 0.35$. The contour lines encircle the $68 \%$ and $95 \%$ confidence levels, respectively. The bottom panel shows the ratio between the observed concentration value and the value predicted by the CLASH-derived c-M relation for each CLASH cluster. The red line shows the median of this ratio for all clusters and the pink area defines the interval between its first and third quartile.

Here, $A$ is the concentration of a halo at the pivot mass and redshift, $B$ the redshift dependence of the concentration and $C$ the dependence on halo mass.

Our data used in the fit contain errors in both mass and concentration, and we expect an intrinsic scatter about the mean relation. Despite this, unbiased estimates of the parameters of the relation can be determined using a likelihood method (e.g., Kelly 2007). In analogy to Hoekstra et al. (2012) and Gruen et al. (2013), we write the likelihood with an additional term that includes the intrinsic scatter as

$$
\begin{aligned}
-2 \ln \mathcal{L} & =\sum_{i} \ln \left(s_{i}^{2}\right)+\left(\frac{\ln \left(c_{i}\right)-\ln \left(c\left(M_{i}, z_{i}\right)\right)}{s_{i}}\right)^{2} \\
s_{i}^{2} & =C^{2} \sigma_{\ln M, i}^{2}+\sigma_{\ln c, i}^{2}+\sigma_{\ln c, \text { int }}^{2},
\end{aligned}
$$

where we use the single-parameter ln-normal measurement uncertainties of mass and concentration $\sigma_{\ln M, i}$ and $\sigma_{\ln c, i}$, an intrinsic ln-normal scatter of concentration $\sigma_{\ln c \text {,int }}$ and Equation (25) as $c\left(M_{i}, z_{i}\right)$, with a sum over all clusters $i$. The likelihood is a function of both the parameters $A, B, C$ and $\sigma_{\ln c \text {,int }}$. For our measurements, it is maximized by $A=3.66 \pm 0.16$, $B=-0.14 \pm 0.52$, and $C=-0.32 \pm 0.18$, where the errors are close to uncorrelated. The intrinsic scatter is consistent with zero at a $1 \sigma$ upper limit of $\sigma_{\ln c \text {,int }}=0.07$.

The results can be summarized as follows.

1. The concentration at the mean mass and redshift of the CLASH sample is constrained at the 5\% level. We detect an indication of a negative slope of concentration with mass. The sign of this slope is in agreement with theoretical expectations (Duffy et al. 2008; Bhattacharya 
et al. 2013), although one has to keep in mind that our measurement has a very low significance of $\sim 1.5 \sigma$ due to our limited dynamic range in mass.

2. Due to the limited dynamic range, our data allow no conclusion on the dependence of concentration on redshift. The theoretical expectation here is to find a negative dependence on redshift from the combined effect of density at the formation time and mass growth (e.g Navarro et al. 1997; Duffy et al. 2008).

To confirm our result with another $\mathrm{c}-\mathrm{M}$ analysis, which is of course not fully independent but different in its methodology, we overplot in Figure 9 the c-M contours at the $68 \%$ and $95 \%$ confidence levels from Umetsu et al. (2014). These contours derive from the stacked weak-shear analysis of 16 CLASH Xray selected clusters. Although the stacked result, which refers to a redshift of $z \simeq 0.35$, lies slightly above the value from our relation, it is in excellent agreement with our results given the uncertainties in both analyses.

\subsection{Comparison with Results from the Literature}

We choose the relations of Duffy et al. (2008) (hereafter D08) and Bhattacharya et al. (2013) (hereafter B13) for our comparison to the CLASH data.

D08 used a set of three $N$-body simulation runs with a comoving box size of 25,100 , and $400 \mathrm{Mpc} / \mathrm{h}$, respectively. All runs adopted a WMAP5 cosmology (Komatsu et al. 2009) and provided a total mass-range of $10^{11}-10^{15} M_{\odot} / h$. In addition, D08 also defined a relaxed sub-sample, with the criterion that the separation between the most bound halo particle and the center of mass of the halo is smaller than $0.07 r_{\text {vir }}$, which was formerly identified as one efficient way of selecting relaxed halos (Neto et al. 2007).

B13 worked with a set of four cosmological boxes ranging in co-moving box size from $128-2000 \mathrm{Mpc} / \mathrm{h}$. Also B13 splits their sample into a full and a relaxed subset, where the relaxed one is defined by the same criterion as in D08. Apart from the larger cosmological boxes, the main difference between D08 and $\mathrm{B} 13$ is the cosmological background model, which resembled a WMAP7 (Komatsu et al. 2011) cosmology in the case of B13 and the larger box size.

\subsection{1. $c-M$ Relation of the Full Samples}

First, we compare the CLASH data set to the full sample c$\mathrm{M}$ relations of D08 and B13. As one can see from visual inspection of Figure 10 already, there is good agreement between the CLASH data and the theoretical c-M relations derived from the simulations.

To statistically quantify the agreement we calculate the ratio $c_{\mathrm{obs}} / c_{\mathrm{sim}}$ as a function of cluster redshift. This ratio for each data point is shown in the bottom panel of Figure 10. Next, we calculate the mean, standard deviation, first, second (median) and third quartiles of all these ratios and report them in Table 8. The median is also shown as horizontal pink line in the bottom panel of Figure 10 with the error range defined by the first and third quartiles. As a last test we perform a Pearson's $\chi^{2}$ test, with the null hypothesis that the theoretical $\mathrm{c}-\mathrm{M}$ relation is a good fit to our data and report the $p$-value in Table 8. All the analysis components, described in this paragraph shall serve as the prototype for all following comparisons between our data and $\mathrm{c}-\mathrm{M}$ relations from simulations. To quantify how well we can expect the data and $\mathrm{c}-\mathrm{M}$ relation to agree, we show in the

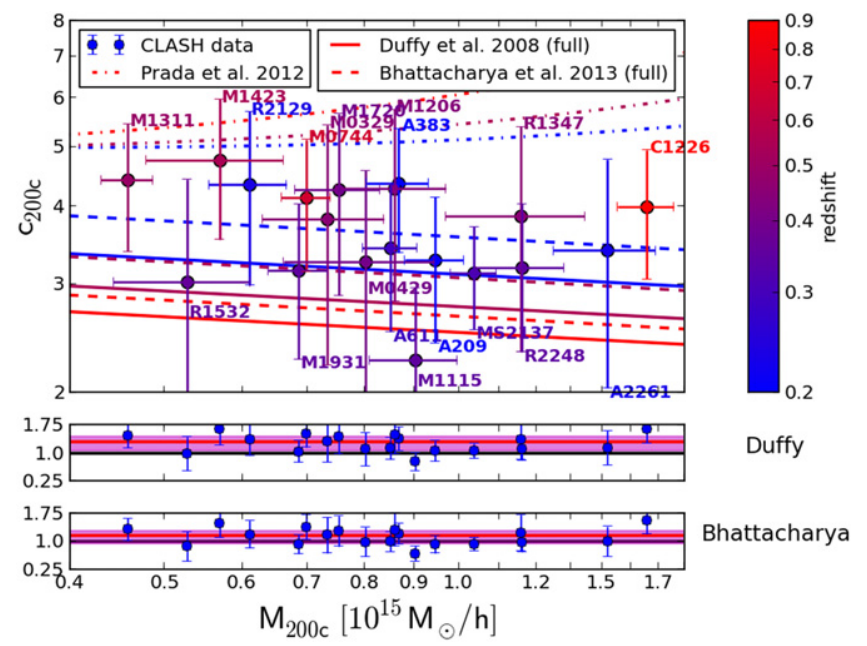

Figure 10. Comparison between CLASH clusters and c-M relations from the literature. This figure is identical in its structure to Figure 9. The lines indicate the $\mathrm{c}-\mathrm{M}$ relations for the full samples of D08, B13, and P12. The bottom panels show the ratio of the observed and the simulation-based concentration, together with the sample median of this ratio and its quartiles.

Table 8

Goodness-of-fit: CLASH Compared to Literature Samples

\begin{tabular}{lllllll}
\hline \hline Reference & $\left\langle c_{\text {obs }} / c_{\text {sim }}\right\rangle^{\mathrm{a}}$ & $Q_{2}{ }^{\mathrm{b}}$ & $Q_{1}{ }^{\mathrm{c}}$ & $Q_{3}{ }^{\mathrm{d}}$ & $\chi^{2}$ & $p$-value \\
\hline CLASH c-M & $1.02 \pm 0.17$ & 1.01 & 0.94 & 1.14 & 7.6 & 0.94 \\
D08 (full) & $1.26 \pm 0.24$ & 1.31 & 1.07 & 1.45 & 15.3 & 0.43 \\
B13 (full) & $1.12 \pm 0.23$ & 1.16 & 0.94 & 1.29 & 11.4 & 0.72 \\
D08 (relaxed) & $1.11 \pm 0.21$ & 1.15 & 0.95 & 1.27 & 10.1 & 0.81 \\
B13 (relaxed) & $1.08 \pm 0.23$ & 1.12 & 0.91 & 1.24 & 11.3 & 0.73 \\
\hline
\end{tabular}

\footnotetext{
The mean of $c_{\mathrm{obs}} / c_{\mathrm{sim}}$ for the full cluster sample.

$\mathrm{b}$ The second quartile or median.

c The first quartile $(25 \%)$.

$\mathrm{d}$ The third quartile $(75 \%)$.
}

very top of Table 8 the comparison to the $\mathrm{c}-\mathrm{M}$ relation which we derived in Section 5.1 from the CLASH data itself.

Finally, Figure 10 also shows the c-M relation of Prada et al. (2012) since it is widely used in the literature. One can easily see that there is a discrepancy between this relation and the CLASH results, especially when the good agreement with the D08 and B13 relations is considered. However, we refer to Meneghetti \& Rasia (2013) which argues that a direct comparison in the c-M plane is not a meaningful comparison in the case of the Prada et al. (2012) relation.

\subsection{2. $c-M$ Relation for the Relaxed Samples}

Since the CLASH clusters were selected to represent a more relaxed sample of clusters than former studies, we expect a much higher level of agreement when comparing to the relaxed subsets of the simulations. The visual comparison is shown in Figure 11, together with the statistical assessment in Table 8. We indeed find that in the case of D08 a 31\% difference between simulation and observation is reduced to $15 \%$, although the two comparisons agree within their error bars as also eported in Table 8 . Note that the change from the full to the relaxed sample $\mathrm{c}-\mathrm{M}$ relation in the work of B13 is only marginal (from $16 \%$ difference to $12 \%$ ), although the same relaxation criterion was applied as in D08. This might either be caused by the different cosmology used in the two simulations 


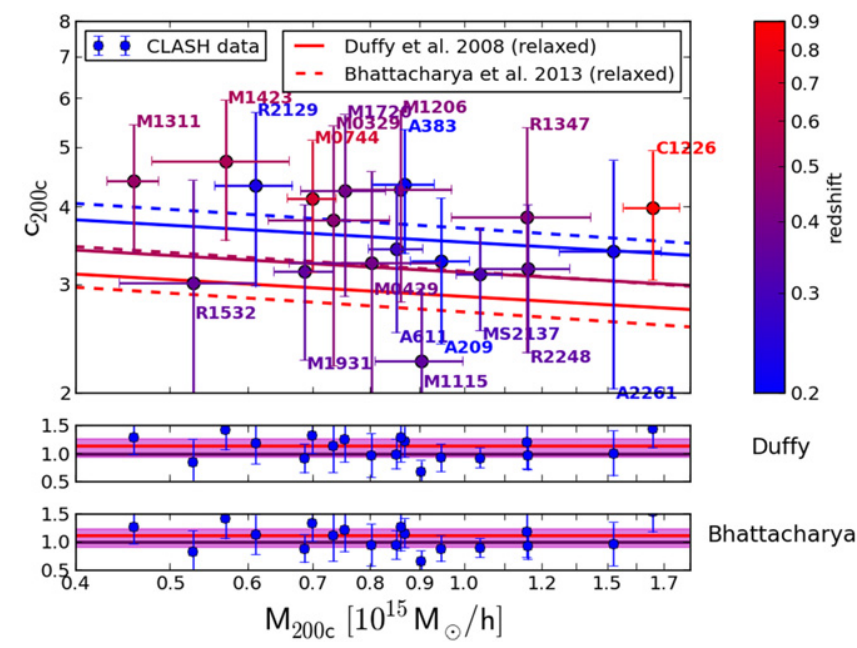

Figure 11. This figure is identical to Figure 10 but shows the $\mathrm{c}-\mathrm{M}$ relations derived from the relaxed samples of D08 and B13.

or might relate to the much bigger set of clusters in B13 and the increased statistical power of the sample.

\section{C-M ANALYSIS WITH A TAILORED SET OF SIMULATIONS}

In the preceding analysis we ignored the fact that we derive our NFW fits from a lensing reconstruction which sees the clusters in projection and we have not properly accounted for the CLASH selection function. We aim at eliminate these shortcomings by using our own set of simulations, where we have full control over the selection of our halo sample and the way in which masses and concentrations are derived from the simulations.

In our companion paper Meneghetti et al. (2014) (hereafter M14) we use a set of 1419 cluster-sized halos from the MUSIC-2 sample (Sembolini et al. 2013). These halos were found in the $1 \mathrm{Gpc}$ MultiDark cosmological simulation (Klypin et al. 2011; Riebe et al. 2013) which was run with a best-fit WMAP7+BAO+SNI cosmology $\left(\Omega_{M}=0.27, \Omega_{\Lambda}=\right.$ $0.73 h=0.7)$. Starting from the large cosmological box with coarse particle mass resolution, the zoom-technique (Klypin et al. 2001) was applied to run re-simulations of the halos of interest with added non-radiative gas physics. This comprehensive set of clusters spans a mass-range between $2 \times 10^{14} M_{\odot} / h-2 \times 10^{15} M_{\odot} / h$ at $z=0$ and is available at four different redshifts $(0.25,0.33,0.43,0.67)$. More details on this set of numerically simulated clusters are given in M14 and J. Vega et al. (2014, in preparation).

\subsection{Analysis in $3 D$}

We measure masses and concentrations of the halos in our simulated sample in a standard way by counting particles in radial bins around the halo center and by assigning a mean density to each bin. The innermost radial bin in this scheme is defined by the spatial resolution of the underlying zoom simulations and the outermost radial bin refers to $r_{200 c}$ of the halo. We fit a NFW profile to the decadic logarithm of the density as described in more detail in Ludlow et al. (2014) and M14. To the measured masses and concentrations of each halo and at all available redshifts we fit a $\mathrm{c}-\mathrm{M}$ relation
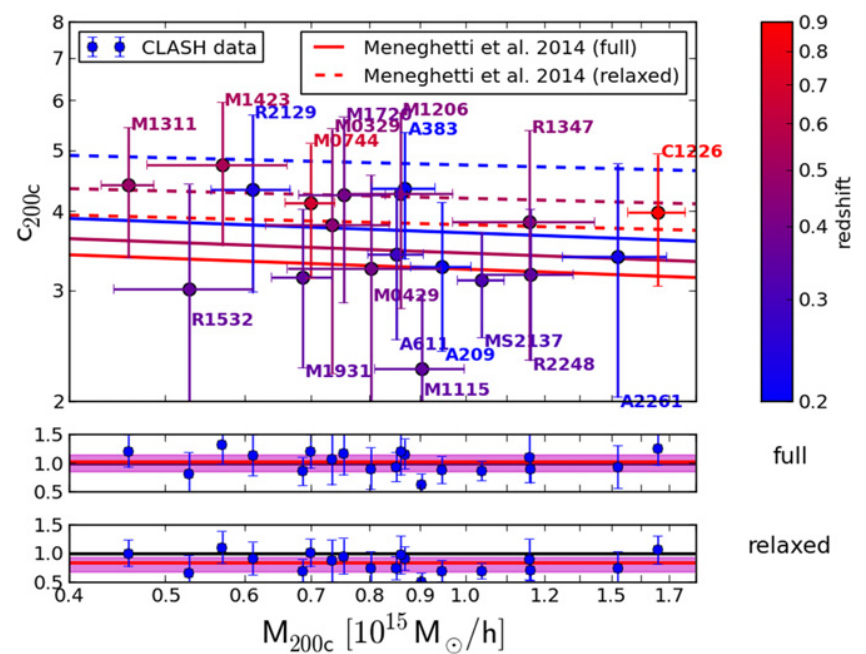

full

relaxed

Figure 12. Comparison between CLASH and a tailored set of c-M relations from numerical simulations. This figure is identical in its structure to Figure 10 and shows the comparison between the CLASH data and the analysis of the simulations by M14 in 3D.

Table 9

Goodness-of-fit: Meneghetti et al. 2014

\begin{tabular}{lccccrc}
\hline \hline Sample & $\left\langle c_{\text {obs }} / c_{\text {sim }}\right\rangle$ & $Q_{2}$ & $Q_{1}$ & $Q_{3}$ & \multicolumn{1}{c}{$\chi^{2}$} & $p$-value \\
\hline 3D full & $1.00 \pm 0.18$ & 1.03 & 0.86 & 1.15 & 9.5 & 0.85 \\
3D relaxed & $0.80 \pm 0.16$ & 0.84 & 0.68 & 0.93 & 29.4 & 0.01 \\
2D full & $1.03 \pm 0.19$ & 1.06 & 0.89 & 1.09 & 9.2 & 0.87 \\
2D relaxed & $0.86 \pm 0.16$ & 0.88 & 0.73 & 0.98 & 32.1 & 0.01 \\
2D SL & $0.91 \pm 0.19$ & 0.93 & 0.78 & 1.03 & 18.0 & 0.26 \\
2D X-ray & $0.94 \pm 0.20$ & 0.96 & 0.80 & 1.06 & 16.1 & 0.38 \\
\hline
\end{tabular}

Note. The column explanations are identical to Table 8 .

following the parametrization of Duffy et al. (2008), adapted to the mass and redshift range of the simulations.

To define a limiting case we construct a strictly relaxed subset $^{38}$ of our simulated sample, by applying all three relaxation criteria of Neto et al. (2007). In addition to the already mentioned ratio of center of mass and virial radius, this includes also a constraint on the halo's substructure mass fraction $f_{\text {sub }}<0.1$ and the restriction that the virial ratio must obey $2 T /|U|<1.35$. For complete definitions of $f_{\text {sub }}, T$ and $U$ see Neto et al. (2007). This selection reduces the number of halos in this strictly relaxed subset to $15 \%$ of the original full sample. Please note that this relaxation criterion is indeed more restrictive than the one used by D08 and B13 which only obeyed the center of mass constraint. The $\mathrm{c}-\mathrm{M}$ relations for both the full and the relaxed sample are shown in Figure 12.

We summarize the quantitative comparison to our observed CLASH results in Table 9. We find excellent agreement between our observed data and the full sample of M14, very similar to the findings of B13. It is indeed reassuring that our baseline $\mathrm{c}-\mathrm{M}$ relation derived from the full set of simulated clusters and analyzed with standard profile-fitting techniques gives a very similar result to B13 since most of our sample clusters were selected from the same parent cosmological box. The picture changes however, when we turn our attention to the strictly relaxed sample of M14, as can be seen in Figure 12 and Table 9. On average, the concentrations of the CLASH sample

\footnotetext{
${ }^{38}$ This is defined as the "super-relaxed" sample in M14.
} 


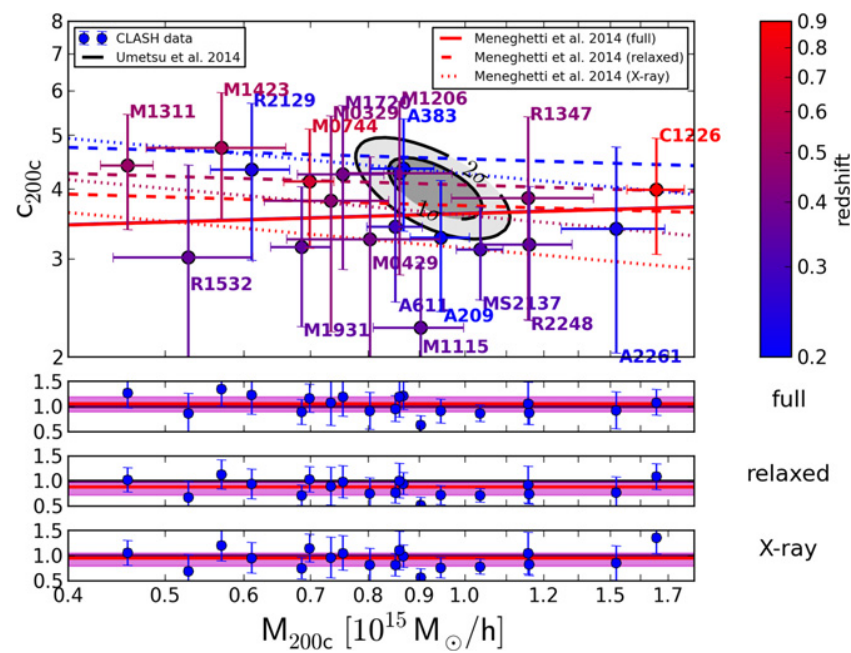

Figure 13. c-M comparison in $2 \mathrm{D}$. This figure is identical to Figure 12, but shows the comparison between different $\mathrm{c}-\mathrm{M}$ relations, based on different halo subsets from M14 in 2D. In addition, we overlay again the c-M likelihood contours from Umetsu et al. (2014).

are underestimated by about $15 \%$ and the associated $p$-value drops from 0.85 in the full to about 0.01 in the relaxed sample. This is in some tension with the results seen for D08 and B13, but we remind the reader that the selection criteria we adopt differ from those in D08 and B13. Specifically, we adopt all three criteria as used by Neto et al. (2007) to create the limiting case of a strictly relaxed sample, while D08 and B13 used a less strict definition of relaxation based on only one of these criteria.

\subsection{Analysis in $2 D$}

One aspect of our analysis may introduce substantial biases, namely that we assume spherical symmetry while fitting a 3D radial profile to our projected data coming from a lensing reconstruction. Several solutions to work around this issue have been proposed, e.g., by using X-ray and SZ data to gain information on the 3D shape of the density profile (see, e.g., Mahdavi et al. 2007; Corless et al. 2009; Morandi et al. 2010, 2012; Sereno et al. 2013). In this work we choose a different approach by also analyzing our simulated data in projection and by making the same assumption of spherical symmetry when deriving the density profiles of the simulated halos.

We perform the projection for each of our halos in the full sample by projecting all simulation particles in a box with $6 \mathrm{Mpc} / \mathrm{h}$ sides around the halo center. We chose 100 randomly selected lines of sight to obtain many realizations of the same halo, thereby increasing the statistical power of our sample. From the projected particle density we derive convergence maps, bin them azimuthally around the halo center and fit a NFW profile to the binned data under the assumption of spherical symmetry. For more details we refer the interested reader to M14 and Vega et al. (2014, in preparation). Also for this 2D case, we define a strictly relaxed sample as limiting case following the criteria outlined in Section 6.1.

The results of the comparison to these 2D c-M relations with the CLASH data can be seen in Figure 13. By applying the same statistical tests we find an excellent agreement with the full 2D sample of M14. When evaluating the median of the ratio between the two, the observed concentrations are only $6 \%$ higher than in the simulated sample which is now free of the projection bias, although the scatter is large. However, when restricting ourselves to the strictly relaxed clusters the $2 \mathrm{D} \mathrm{c}-\mathrm{M}$ relation is in tension with observations. The $p$-value drops from 0.87 to 0.01 and the difference in the median concentration ratio increases to $12 \%$. The situation improves significantly to only $7 \%$ overestimation in the concentration ratio and a $p$-value of 0.26 once we pick only those simulated clusters which are able to produce strong-lensing features by demanding that the cluster produces a critical line (comp. Equation (10)). This selection is appropriate since all but one CLASH cluster allowed us to identify strong lensing features. However, the observational data is clearly in tension with a simulated cluster sample selected after the three relaxation criteria of Neto et al. (2007) and which is analyzed in 2D. This highlights the importance of halo selection and the necessity to properly account for the CLASH selection function.

\subsubsection{X-Ray Selection of CLASH Clusters}

As is pointed out in Postman et al. (2012a), the CLASH Xray selected sample was designed to have a mostly regular Xray morphology. Therefore, we perform yet another selection from our M14 cluster sample, mimicking the CLASH X-ray selection. As pointed out in Section 6 of M14, the selection based on X-ray regularity is related to but not identical to a selection based on halo relaxation. The X-ray selection is possible with the help of the X-MAS simulator (Rasia et al. 2008; Meneghetti et al. 2010b; Rasia et al. 2012) which produces simulated X-ray observations from a numerically simulated halo. We conFigure X-MAS to reproduce the X-ray observations (Ebeling et al. 2007; Allen et al. 2008; Cavagnolo et al. 2008; Maughan et al. 2008; Mantz et al. 2010) according to which the CLASH clusters were selected. Using this set of simulated X-ray images we apply the very same selection criteria which were used to select the CLASH X-ray selected clusters. For a more detailed description of these criteria and the selection process see M14.

This CLASH-like, X-ray selected sample in 2D is the one simulated sample which comes closest to the real CLASH clusters, both with respect to the selection criteria and the analysis method. The comparison between the $\mathrm{c}-\mathrm{M}$ relation of this sample and the observed CLASH clusters shows indeed significant improvement over the limiting case of the fully relaxed sample in the last section. The qualitative agreement between the data points and the X-ray selected $\mathrm{c}-\mathrm{M}$ relation in Figure 13 is quite obvious. The median concentration ratio shows that the observed CLASH concentrations are only $4 \%$ lower than the ones from the X-ray selected simulation sample and the $p$-value 0.38 indicates no strong tension between the two samples (compare Table 9). Finally, we calculate the $\Delta \chi^{2}$ value for the fits of the CLASH c-M relation from Section 5.1 and the X-ray selected $\mathrm{c}-\mathrm{M}$ relation and find that the two relations agree at the $90 \%$ confidence level.

\subsection{Individual CLASH Clusters in Our Simulated Sample}

As the final analysis in this work we now select close matches to individual CLASH clusters out of our 2D set of simulated halos. We do this in order to gather additional confirmation that our specific way of selecting CLASH clusters from a numerical simulation is sufficiently accurate to characterize the CLASH selection function. We find simulated 


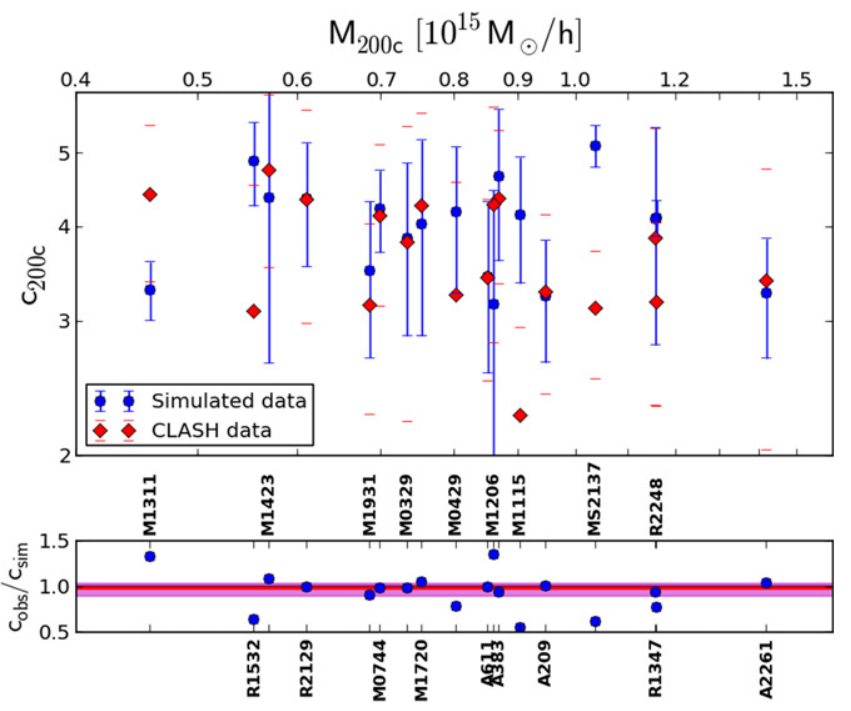

Figure 14. Distribution of observed and simulated concentrations for $18 \mathrm{X}$-ray selected CLASH clusters. The blue points show the expected concentration for each CLASH cluster as it is derived from all halo projections which fulfill our CLASH X-ray selection criteria for that specific halo. The red points show the observational equivalent. In the bottom panel we show the ratio between the two concentration values, together with the median of the ratio sample in red. The pink error band is defined by the first and third quartile of this sample.

counterparts to individual CLASH clusters by means of a regularity metric defined in Section 4 of M14. After all matching projections have been found for a single CLASH cluster, we calculated the expected concentration by a weighted average over the concentrations of these projections (see Section 7 of M14 for details). In the course of this analysis we had to drop CLJ1226 because no match was found in our simulated set. The system is very massive and sits at high redshift which would require a larger simulation to find an equivalent. ${ }^{39}$ We show the findings of the remaining 18 systems in Figure 14, where we compare the expected concentration for each individual simulated CLASH-like cluster with the findings from observations. All but two points overlap within the $1 \sigma$ error bars and the ratio between observed and simulated concentrations for all CLASH clusters is close to a perfect match with the median of $c_{\text {obs }} / c_{\text {sim }} Q_{2}=0.99_{-0.09}^{+0.05}$ where the error margins are defined by the first and third quartiles of the sample. The fact that the selection of individual CLASH clusters shows good agreement between predicted and observed concentrations gives us some confidence that we are indeed able to characterize the CLASH selection function by means of X-ray morphology.

We provide a general statistical summary of the distribution of simulated concentrations in Table 10 and we conclude our comparison to the simulations of M14 with a two-sample statistical analysis. We perform a Kolmogorov-Smirnov test and find a $p$-value of 0.75 , again showing no indication for tension in the null hypothesis that the observed and simulated data have the same parent distribution of $\mathrm{c}-\mathrm{M}$ values.

\section{CONCLUSIONS}

The HST multi-cycle treasury program CLASH was in part designed to shed light on the dark matter density profile of

\footnotetext{
39 An even more massive system at similar redshift has been observed (e.g., Menanteau et al. 2012; Jee et al. 2014b).
}

Table 10

General Properties of Concentration Samples

\begin{tabular}{lccccccc}
\hline \hline Sample & Mean & SD & $Q_{2}$ & $Q_{1}$ & $Q_{3}$ & Min & Max \\
\hline Observed data & 3.65 & 0.65 & 3.43 & 3.18 & 4.26 & 2.26 & 4.75 \\
Simulated data & 3.87 & 0.61 & 3.76 & 3.62 & 3.93 & 3.07 & 5.68 \\
\hline
\end{tabular}

Note. The column explanations are identical to Table 8.

galaxy clusters by combining the enormous resolving power of the $H S T$ with wide-field Subaru imaging. The CLASH X-ray selected sample of galaxy clusters was specifically selected to have a mostly undisturbed X-ray morphology, suggesting that this sub-sample represents an undisturbed and unbiased set of clusters in terms of their density profile. This choice was made since former studies of lensing clusters with exquisite data quality were inconsistent with the predictions of $\Lambda \mathrm{CDM}$, and selection effects were thought to be a possible cause of this disagreement.

In this work we applied advanced lensing reconstruction techniques to this CLASH data set. Our reconstructions combines weak and strong lensing to fully exploit the lensing data provided by the CLASH program. With the help of adaptively refined grids, we achieve a non-parametric reconstruction of the lensing potential over a wide range of scales, from the inner-most strong-lensing core of the system at scales $\sim 10 \mathrm{kpc}$ out to the virial radius at $\sim 2 \mathrm{Mpc}$. This is the first time that such a multi-scale reconstruction using weak and strong lensing has been performed for such a large sample of clusters. Fits to the surface-mass density profiles provide masses and concentrations for 19 massive galaxy clusters.

In order to have full control over the selection function of halos and in order to avoid possible biases introduced by the tri-axial structure of high-mass halos, we also derive c-M relations from a new, unique set of simulated halos. These simulations allow us to make specific choices in our selection and analysis, providing a much closer match to real observations. While simulations are usually analyzed in 3D we perform a purely $2 \mathrm{D}$ analysis in projection, as this is the only option for the observed lensing data. We apply different selection functions to the simulations, including a selection based on the X-ray morphology of realistic X-ray images of our hydrosimulations. This sample obeys the selection criteria of CLASH. This is of great importance since the selection of a cluster from a numerical simulation based on X-ray regularity, like in the case of CLASH, relates to but is not identical to a selection based on relaxation parameters only. The details of this selection function are studied in much more detail in another CLASH paper by Meneghetti et al. (2014). For the Xray selected 2D sample we find excellent agreement between simulations and observations. Observed concentration are on average only $4 \%$ lower than in simulations and we find no statistical indication for tension between the simulated and observed data set. This detailed comparison between observations and simulations in 2D, with full consideration of the underlying selection function is unique and gives us great confidence in the results, which are a confirmation of the $\Lambda \mathrm{CDM}$ paradigm, at least in the context of a $\mathrm{c}-\mathrm{M}$ relation of cluster-sized halos.

From fitting a c-M relation to the CLASH data directly we find our concentrations distributed around a central value of $c_{200 c} \simeq 3.7$ with a mild negative slope in mass at the $1 \sigma$-level. 


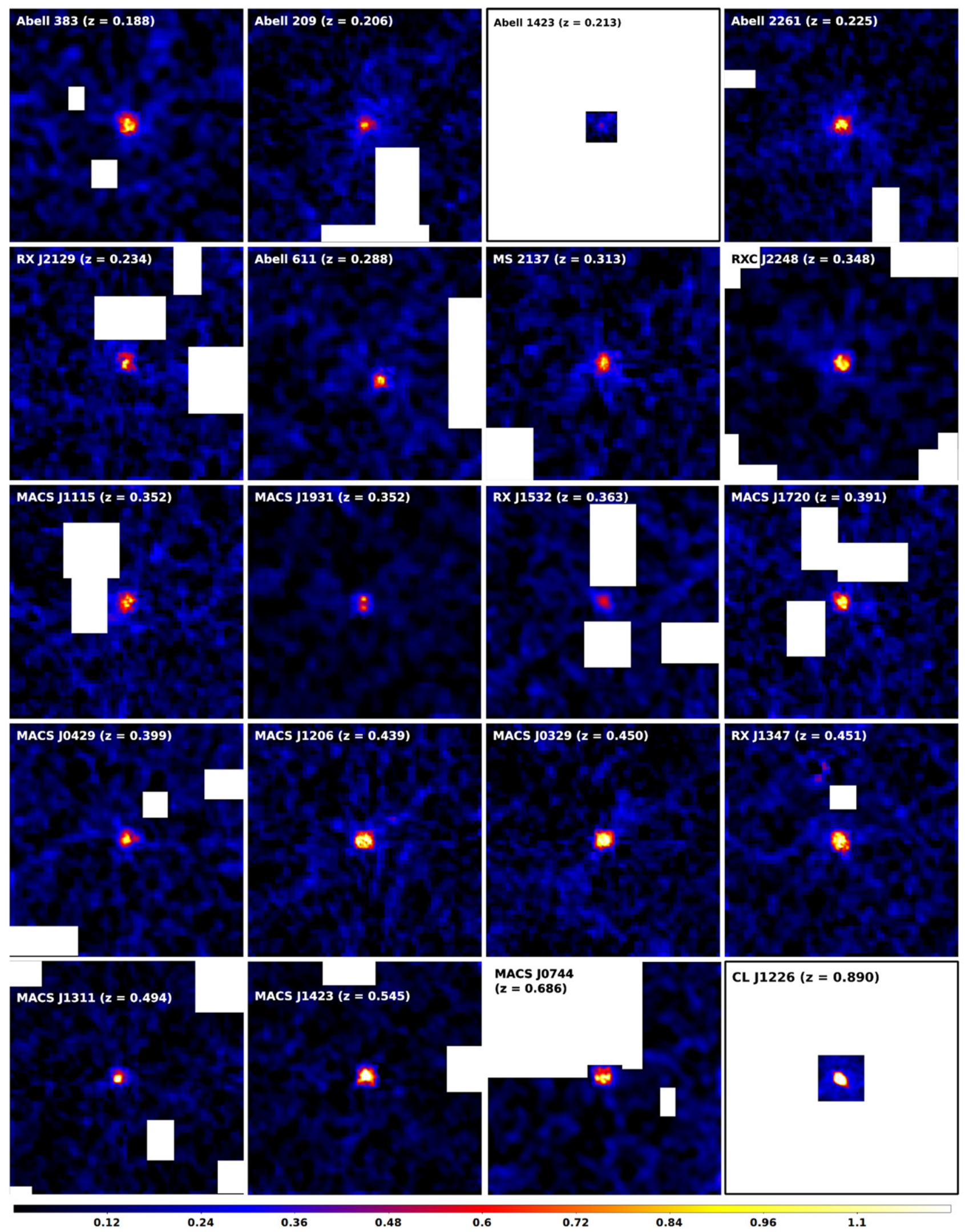

Figure 15. Convergence maps for $20 \mathrm{X}$-ray selected CLASH clusters. The field size for the map of Abell 1423 is $200^{\prime \prime}$, for CL J1226 it is $300^{\prime \prime}$, and for Abell 611 it is $1400^{\prime \prime}$. For all other clusters the field size is $1500^{\prime \prime}$. The color coding, together with the colorbar shows the lensing convergence, scaled to a redshift of $z=20,000$. Extended white patches in the convergence maps indicate field masks, usually at the position of bright foreground stars. The orientation of all maps is north is up. 
Abell $383(z=0.188)$

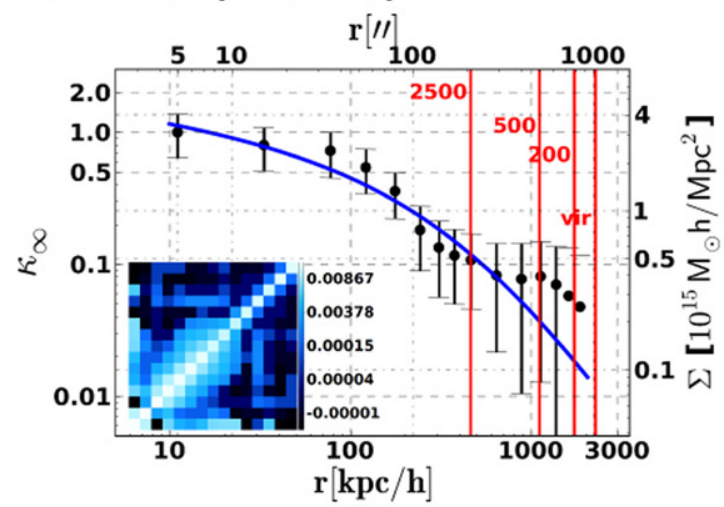

Abell $2261(z=0.225)$

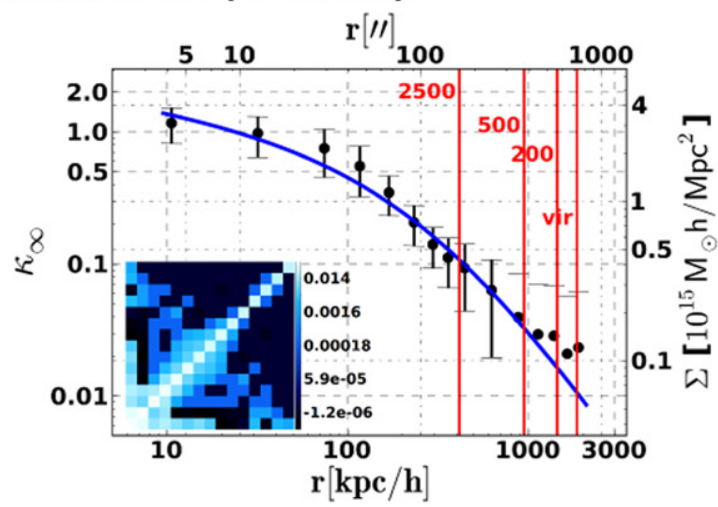

Abell $611(z=0.288)$

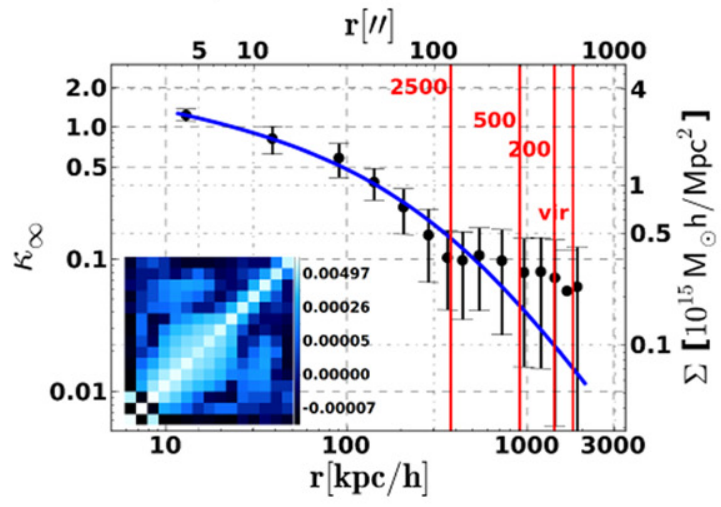

RXC J2248 $(z=0.348)$

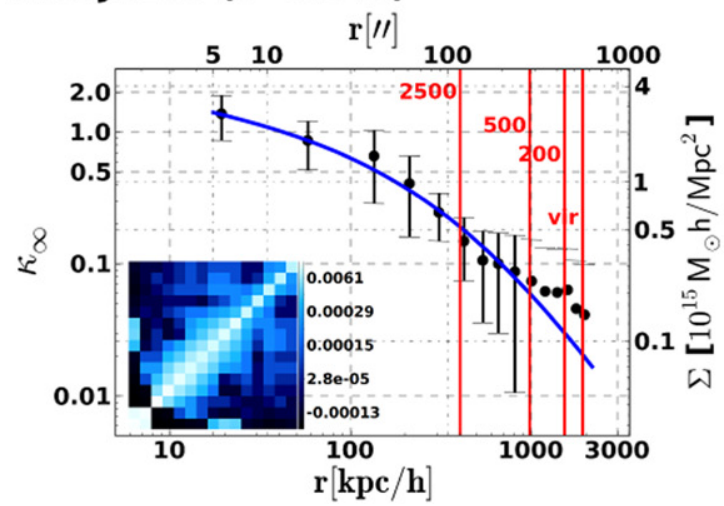

Abell $209(z=0.206)$

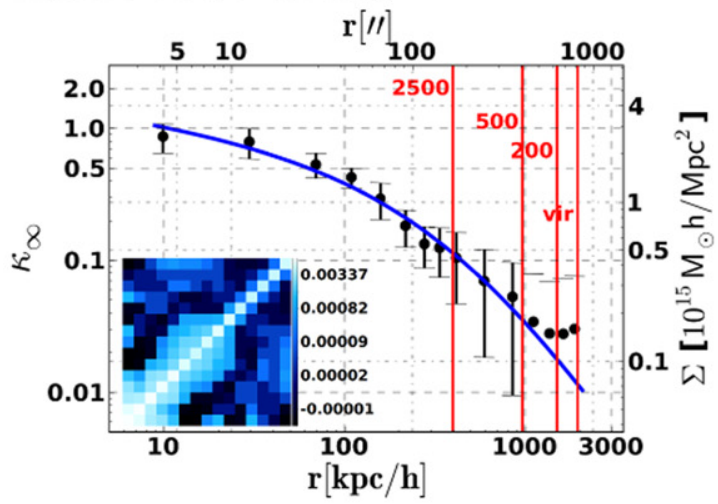

RX J2129 $(z=0.234)$

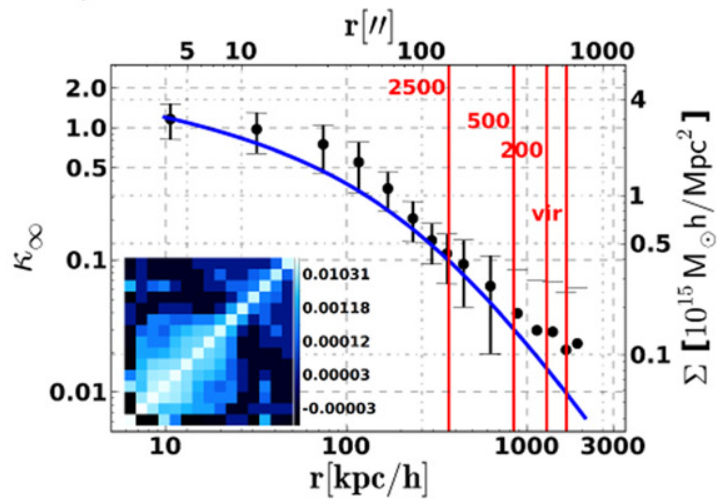

MS $2137(z=0.313)$

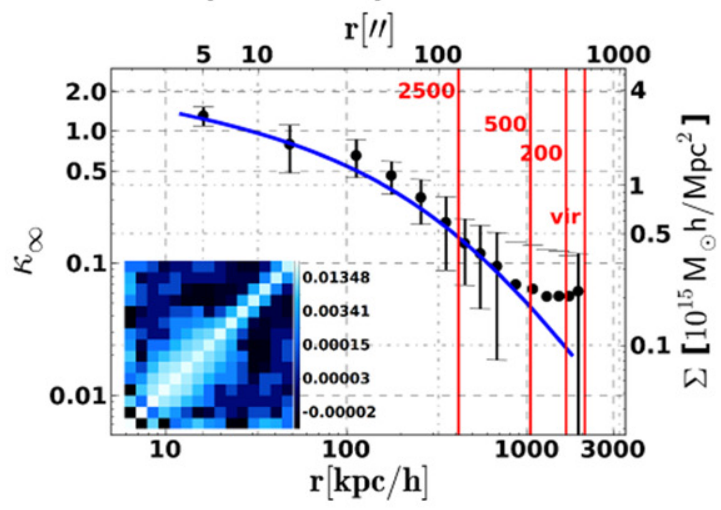

MACS J1115 $(z=0.352)$

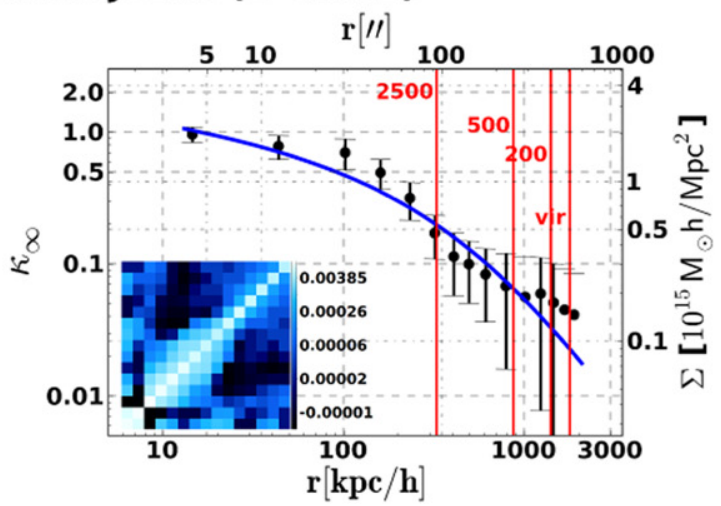

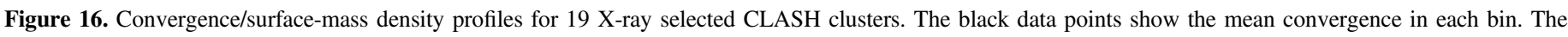

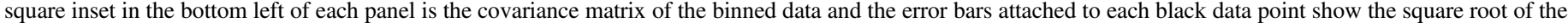

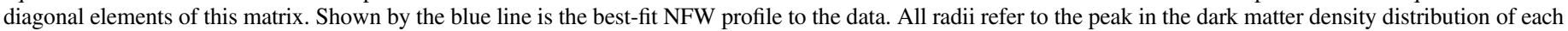
halo as a center. Drawn in red are $r_{2500}, r_{500}$, and $r_{200}$ and the virial radius of the halo. The convergence values are scaled to a source redshift of $z=20,000$. 
MACS J1931 $(z=0.352)$

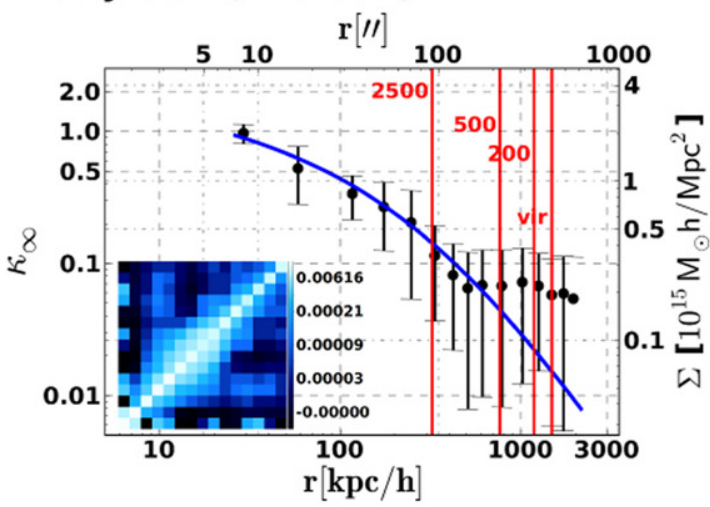

MACS J1720 $(z=0.391)$

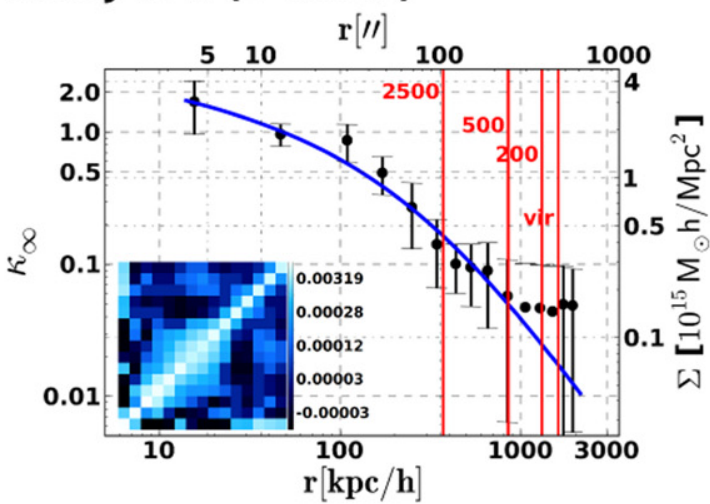

MACS J1206 $(z=0.439)$

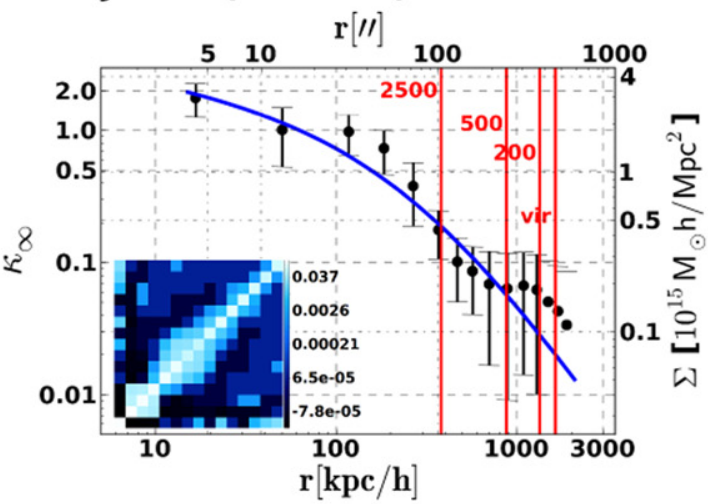

RX J1347 $(z=0.451)$

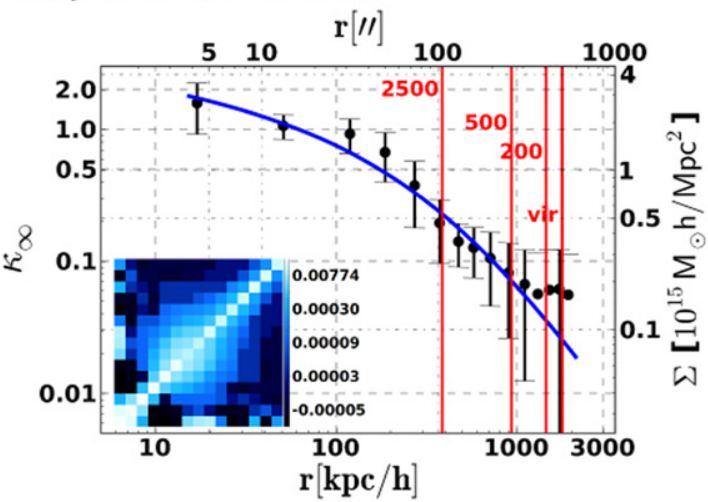

RX J1532 $(z=0.363)$

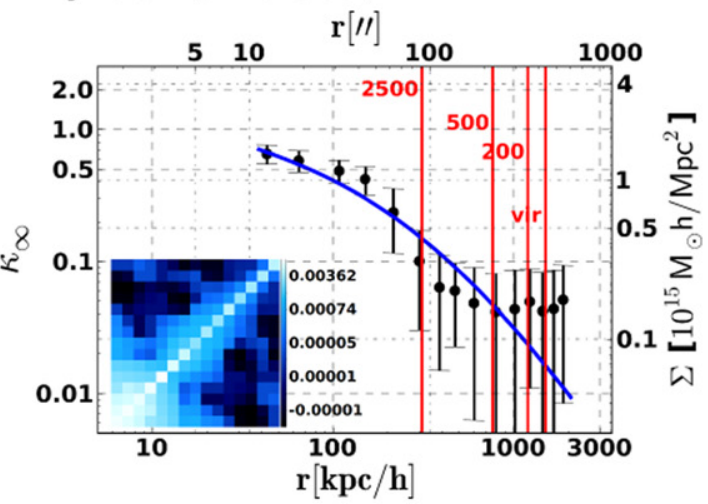

MACS J0429 $(z=0.399)$

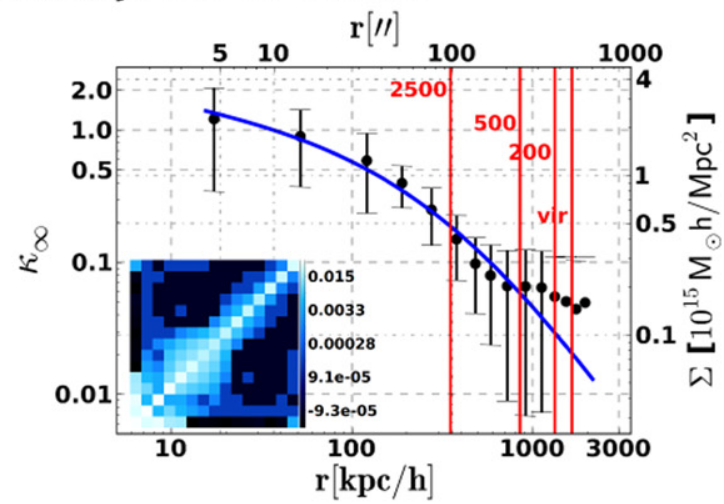

MACS J0329 $(z=0.450)$

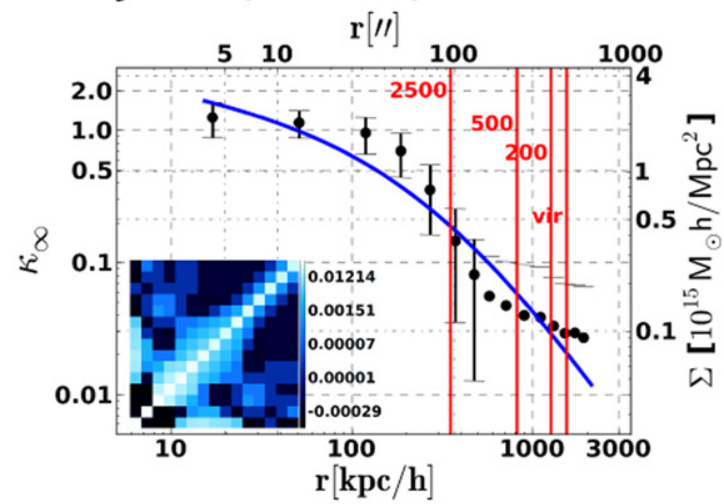

MACS J1311 $(z=0.494)$

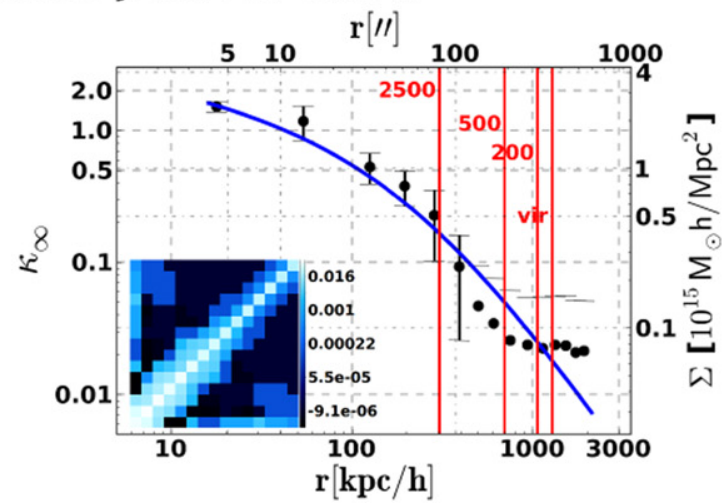

Figure 16. (Continued.) 
MACS J1423 $(z=0.545)$

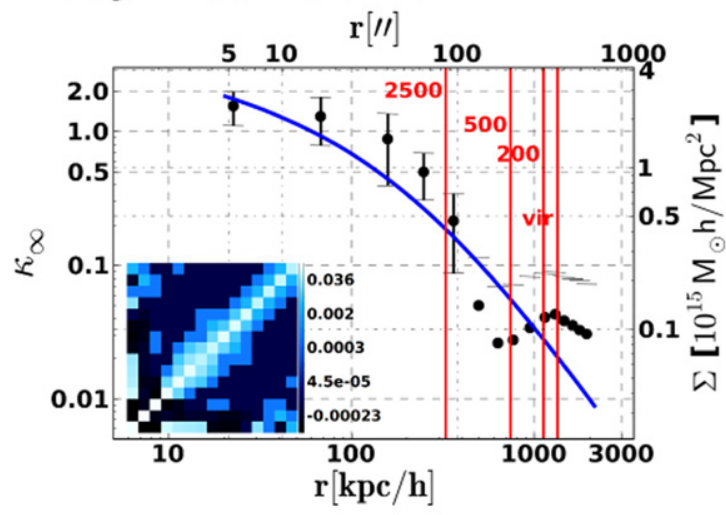

MACS J0744 $(z=0.686)$

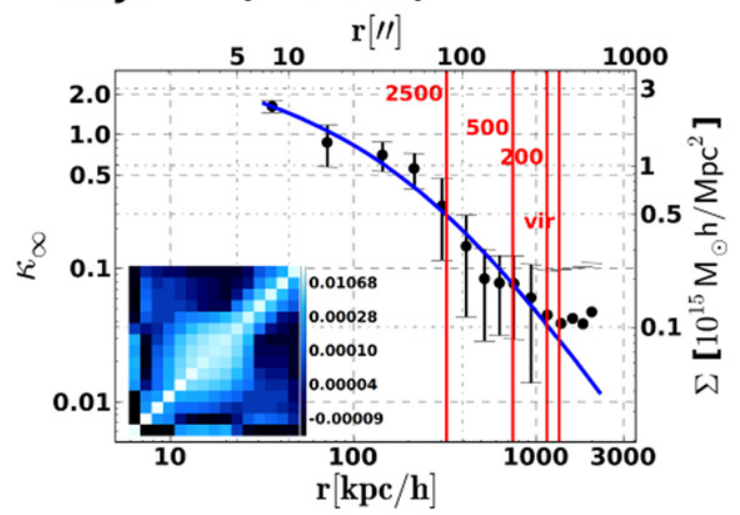

CL J1226 ( $z=0.890)$

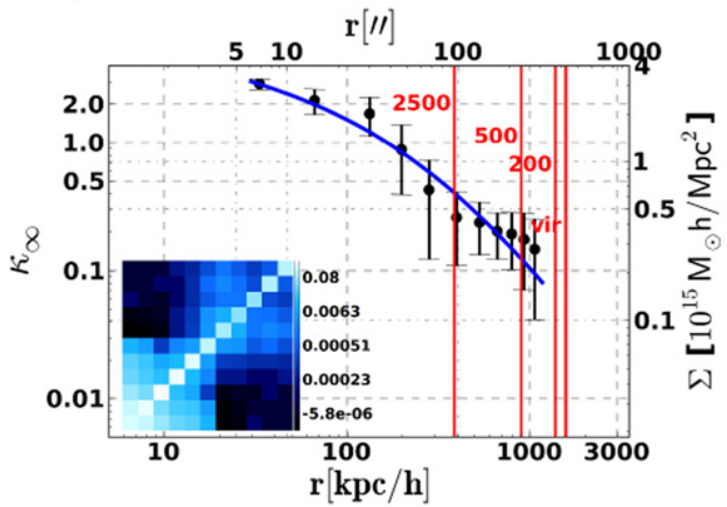

Figure 16. (Continued.)

This $\mathrm{c}-\mathrm{M}$ relation derived from the CLASH data directly agrees with the c-M relation of simulated X-ray selected halos analyzed in projection at the $90 \%$ confidence level. Our comprehensive likelihood analysis shows that we are insensitive to any possible redshift dependence of the $\mathrm{c}-\mathrm{M}$ relation. A larger leverage in redshift would be needed to probe this trend which is suggested by numerical simulations.

We want to highlight the complementary work on CLASH weak lensing and magnification measurements by Umetsu et al. (2014) and the full characterization of the CLASH simulations by Meneghetti et al. (2014). However, due to the exquisite quality of the lensing data used for this analysis, further and more advanced studies will be possible. Ongoing analyses include additional functional forms describing the dark matter distribution, like the generalized NFW or Einasto profiles. Particularly the analysis of inner slopes of the CLASH clusters and the intrinsic scatter of $\mathrm{c}-\mathrm{M}$ relations derived from these profiles will give interesting insights into the physics of dark matter and the role of baryons on cluster scales. Ultimately, one would like to go away from $1 \mathrm{D}$, radial density profiles and describe the full morphology and shape of the dark matter distributions in observations and simulations. Such techniques might indeed prove more powerful in, e.g., distinguishing different particle models of dark matter. The CLASH clusters are clearly the ideal data set to perform such analyses.

The research was in part carried out at the Jet Propulsion Laboratory, California Institute of Technology, under a contract with the National Aeronautics and Space Administration. J. M. has received funding from the People Programme
(Marie Curie Actions) of the European Union's Seventh Framework Programme (FP7/2007-2013) under REA grant agreement number 627288. M. M. thanks ORAU and NASA for supporting his research at JPL and acknowledges support from the contract ASI/INAF I/023/12/0, INFN/PD51, and the PRIN MIUR 20102011 "The dark universe and the cosmic evolution of baryons: from current surveys to Euclid." K. U. acknowledges support from the National Science Council of Taiwan (grant NSC100-2112-M-001-008-MY3) and from the Academia Sinica Career Development Award. Support for A. Z. is provided by NASA through Hubble Fellowship grant \#HST-HF-51334.01 A awarded by STScI. D. G., S. S. and P. R. were supported by SFB Transregio 33 "The Dark universe" by the Deutsche Forschungsgemeinschaft (DFG) and the DFG cluster of excellence "Origin and Structure of the universe." This work was supported in part by contract research "Internationale Spitzenforschung II/2-6" of the Baden Württemberg Stiftung. The Dark Cosmology Centre is funded by the DNRF. J.S. was supported by NSF/AST1313447, NASA/ NNX11AB07G, and the Norris Foundation CCAT Postdoctoral Fellowship. E.R. acknowledges support from the National Science Foundation AST-1210973, SAO TM3-14008X (issued under NASA Contract No. NAS8-03060)

\section{REFERENCES}

Abdelsalam, H. M., Saha, P., \& Williams, L. L. R. 1998, MNRAS, 294, 734 Abell, G. O. 1958, ApJS, 3, 211

Abell, G. O., Corwin, H. G., Jr., \& Olowin, R. P. 1989, ApJS, 70, 1

Allen, S. W., Rapetti, D. A., Schmidt, R. W., et al. 2008, MNRAS, 383, 879 Anderson, J., \& Bedin, L. R. 2010, PASP, 122, 1035 
Anderson, L., Aubourg, E., Bailey, S., et al. 2012, MNRAS, 427, 3435

Applegate, D. E., von der Linden, A., Kelly, P. L., et al. 2014, MNRAS, 439, 48

Bacon, D. J., Goldberg, D. M., Rowe, B. T. P., \& Taylor, A. N. 2006, MNRAS, 365, 414

Balestra, I., Vanzella, E., Rosati, P., et al. 2013, A\&A, 559, L9

Bartelmann, M. 2010, CQGra, 27, 233001

Bartelmann, M., Narayan, R., Seitz, S., \& Schneider, P. 1996, ApJL, 464, L115+

Bartelmann, M., \& Schneider, P. 2001, PhR, 340, 291

Becker, M. R., \& Kravtsov, A. V. 2011, ApJ, 740, 25

Benítez, N. 2000, ApJ, 536, 571

Bhattacharya, S., Habib, S., Heitmann, K., \& Vikhlinin, A. 2013, ApJ, 766, 32

Biviano, A., Rosati, P., Balestra, I., et al. 2013, A\&A, 558, A1

Borgani, S., \& Kravtsov, A. 2011, ASL, 4, 204

Boylan-Kolchin, M., Bullock, J. S., \& Kaplinghat, M. 2012, MNRAS, 422, 1203

Bradač, M., Allen, S. W., Treu, T., et al. 2008, ApJ, 687, 959

Bradač, M., Clowe, D., Gonzalez, A. H., et al. 2006, ApJ, 652, 937

Bradač, M., Erben, T., Schneider, P., et al. 2005a, A\&A, 437, 49

Bradač, M., Lombardi, M., \& Schneider, P. 2004, A\&A, 424, 13

Bradač, M., Schneider, P., Lombardi, M., \& Erben, T. 2005b, A\&A, 437, 39

Bridle, S. L., Hobson, M. P., Lasenby, A. N., \& Saunders, R. 1998, MNRAS, 299, 895

Broadhurst, T., Benítez, N., Coe, D., et al. 2005, ApJ, 621, 53

Broadhurst, T., Umetsu, K., Medezinski, E., Oguri, M., \& Rephaeli, Y. 2008, ApJL, 685, L9

Broadhurst, T. J., \& Barkana, R. 2008, MNRAS, 390, 1647

Broadhurst, T. J., Taylor, A. N., \& Peacock, J. A. 1995, ApJ, 438, 49

Bullock, J. S., Kolatt, T. S., Sigad, Y., et al. 2001, MNRAS, 321, 559

Buote, D. A., Gastaldello, F., Humphrey, P. J., et al. 2007, ApJ, 664, 123

Cacciato, M., Bartelmann, M., Meneghetti, M., \& Moscardini, L. 2006, A\&A, 458,349

Cavagnolo, K. W., Donahue, M., Voit, G. M., \& Sun, M. 2008, ApJ, 682, 821

Clowe, D., Bradač, M., Gonzalez, A. H., et al. 2006, ApJL, 648, L109

Coe, D., Umetsu, K., Zitrin, A., et al. 2012, ApJ, 757, 22

Coe, D., Zitrin, A., Carrasco, M., et al. 2013, ApJ, 762, 32

Comerford, J. M., \& Natarajan, P. 2007, MNRAS, 379, 190

Corless, V. L., King, L. J., \& Clowe, D. 2009, MNRAS, 393, 1235

D’Aloisio, A., \& Natarajan, P. 2011, MNRAS, 411, 1628

di Nino, D., Makidon, R. B., Lallo, M., et al. 2008, HST Focus Variations with Temperature, Tech. Rep. 2008-03

Diego, J. M., Tegmark, M., Protopapas, P., \& Sandvik, H. B. 2007, MNRAS, 375,958

Donahue, M., Voit, G. M., Mahdavi, A., et al. 2014, ApJ, 794, 136

Duffy, A. R., Schaye, J., Kay, S. T., \& Dalla Vecchia, C. 2008, MNRAS, 390, L64

Ebeling, H., Barrett, E., Donovan, D., et al. 2007, ApJL, 661, L33

Ebeling, H., Edge, A. C., \& Henry, J. P. 2001, ApJ, 553, 668

Ebeling, H., Edge, A. C., Mantz, A., et al. 2010, MNRAS, 407, 83

Eke, V. R., Navarro, J. F., \& Steinmetz, M. 2001, ApJ, 554, 114

Ettori, S., Donnarumma, A., Pointecouteau, E., et al. 2013, SSRv, 177, 119

Ettori, S., Gastaldello, F., Leccardi, A., et al. 2010, A\&A, 524, A68

Falco, E. E., Gorenstein, M. V., \& Shapiro, I. I. 1985, ApJL, 289, L1

Fedeli, C. 2012, MNRAS, 424, 1244

Fedeli, C., \& Bartelmann, M. 2007, A\&A, 461, 49

Francis, M. J., Lewis, G. F., \& Linder, E. V. 2009, MNRAS, 394, 605

Gao, L., Navarro, J. F., Cole, S., et al. 2008, MNRAS, 387, 536

Gorenstein, M. V., Shapiro, I. I., \& Falco, E. E. 1988, ApJ, 327, 693

Grossi, M., \& Springel, V. 2009, MNRAS, 394, 1559

Gruen, D., Brimioulle, F., Seitz, S., et al. 2013, MNRAS, 432, 1455

Halkola, A., Hildebrandt, H., Schrabback, T., et al. 2008, A\&A, 481, 65

Hanami, H., Ishigaki, T., Fujishiro, N., et al. 2012, PASJ, 64, 70

Hennawi, J. F., Dalal, N., Bode, P., \& Ostriker, J. P. 2007, ApJ, 654, 714

Hoekstra, H., Hartlap, J., Hilbert, S., \& van Uitert, E. 2011, MNRAS, 412, 2095

Hoekstra, H., Mahdavi, A., Babul, A., \& Bildfell, C. 2012, MNRAS, 427, 1298

Host, O. 2012, MNRAS, 420, L18

Ilbert, O., Capak, P., Salvato, M., et al. 2009, ApJ, 690, 1236

Jee, M. J., Ford, H. C., Illingworth, G. D., et al. 2007, ApJ, 661, 728

Jee, M. J., Hoekstra, H., Mahdavi, A., \& Babul, A. 2014a, ApJ, 783, 78

Jee, M. J., Hughes, J. P., Menanteau, F., et al. 2014b, ApJ, 785, 20

Jee, M. J., Mahdavi, A., Hoekstra, H., et al. 2012, ApJ, 747, 96

Jee, M. J., \& Tyson, J. A. 2009, ApJ, 691, 1337

Jouvel, S., Host, O., Lahav, O., et al. 2014, A\&A, 562, A86

Kaiser, N., Squires, G., \& Broadhurst, T. 1995, ApJ, 449, 460
Kelly, B. C. 2007, ApJ, 665, 1489

Kelly, P. L., von der Linden, A., Applegate, D. E., et al. 2014, MNRAS, 439, 28

Killedar, M., Borgani, S., Meneghetti, M., et al. 2012, MNRAS, 427, 533

Kitching, T. D., Balan, S. T., Bridle, S., et al. 2012, MNRAS, 423, 3163

Klypin, A., Kravtsov, A. V., Bullock, J. S., \& Primack, J. R. 2001, ApJ, 554, 903

Klypin, A. A., Trujillo-Gomez, S., \& Primack, J. 2011, ApJ, 740, 102

Koekemoer, A. M., Faber, S. M., Ferguson, H. C., et al. 2011, ApJS, 197, 36

Koekemoer, A. M., Fruchter, A. S., Hook, R. N., \& Hack, W. 2002, in Proc. of the 2002 HST Calibration Workshop, ed. S. Arribas, A. Koekemoer, \& B. Whitmore (Baltimore, MD: STScI), 337

Komatsu, E., Dunkley, J., Nolta, M. R., et al. 2009, ApJS, 180, 330

Komatsu, E., Smith, K. M., Dunkley, J., et al. 2011, ApJS, 192, 18

Korngut, P. M., Dicker, S. R., Reese, E. D., et al. 2011, ApJ, 734, 10

Leauthaud, A., Massey, R., Kneib, J., et al. 2007, ApJS, 172, 219

Lemze, D., Broadhurst, T., Rephaeli, Y., Barkana, R., \& Umetsu, K. 2009, ApJ, 701, 1336

Liesenborgs, J., de Rijcke, S., \& Dejonghe, H. 2006, MNRAS, 367, 1209

Ludlow, A. D., Navarro, J. F., Angulo, R. E., et al. 2014, MNRAS, 441, 378

Mahdavi, A., Hoekstra, H., Babul, A., Balam, D. D., \& Capak, P. L. 2007, ApJ, 668,806

Mandelbaum, R., Rowe, B., Bosch, J., et al. 2014, ApJS, 212, 5

Mantz, A., Allen, S. W., Rapetti, D., \& Ebeling, H. 2010, MNRAS, 406, 1759

Massey, R. 2010, MNRAS, 409, L109

Massey, R., \& Goldberg, D. M. 2008, ApJL, 673, L111

Massey, R., Hoekstra, H., Kitching, T., et al. 2013, MNRAS, 429, 661

Massey, R., Refregier, A., Conselice, C. J., David, J., \& Bacon, J. 2004, MNRAS, 348, 214

Massey, R., Rhodes, J., Ellis, R., et al. 2007, Natur, 445, 286

Maughan, B. J., Jones, C., Forman, W., \& van Speybroeck, L. 2008, ApJS, 174,117

Medezinski, E., Broadhurst, T., Umetsu, K., \& Coe, D. 2008, MPLA, 23, 1521

Medezinski, E., Broadhurst, T., Umetsu, K., et al. 2007, ApJ, 663, 717

Medezinski, E., Broadhurst, T., Umetsu, K., et al. 2010, MNRAS, 405, 257

Medezinski, E., Umetsu, K., Nonino, M., et al. 2013, ApJ, 777, 43

Melchior, P., Suchyta, E., Huff, E., et al. 2015, MNRAS, 449, 2219

Menanteau, F., Hughes, J. P., Sifón, C., et al. 2012, ApJ, 748, 7

Meneghetti, M., Fedeli, C., Pace, F., Gottlöber, S., \& Yepes, G. 2010a, A\&A, 519, A90

Meneghetti, M., Fedeli, C., Zitrin, A., et al. 2011, A\&A, 530, A17

Meneghetti, M., \& Rasia, E. 2013, arXiv:1303.6158

Meneghetti, M., Rasia, E., Merten, J., et al. 2010b, A\&A, 514, A93

Meneghetti, M., Rasia, E., Vega, J., et al. 2014, ApJ, 797, 34

Merten, J. 2014, arXiv:1412.5186

Merten, J., Cacciato, M., Meneghetti, M., Mignone, C., \& Bartelmann, M. 2009, A\&A, 500, 681

Merten, J., Coe, D., Dupke, R., et al. 2011, MNRAS, 417, 333

Monna, A., Seitz, S., Greisel, N., et al. 2014, MNRAS, 438, 1417

Morandi, A., Limousin, M., Sayers, J., et al. 2012, MNRAS, 425, 2069

Morandi, A., Pedersen, K., \& Limousin, M. 2010, ApJ, 713, 491

Navarro, J. F., Frenk, C. S., \& White, S. D. M. 1996, ApJ, 462, 563

Navarro, J. F., Frenk, C. S., \& White, S. D. M. 1997, ApJ, 490, 493

Neto, A. F., Gao, L., Bett, P., et al. 2007, MNRAS, 381, 1450

Newman, A. B., Treu, T., Ellis, R. S., et al. 2013, ApJ, 765, 24

Oguri, M., Bayliss, M. B., Dahle, H., et al. 2012, MNRAS, 420, 3213

Okabe, N., Smith, G. P., Umetsu, K., Takada, M., \& Futamase, T. 2013, ApJL, $769, \mathrm{~L} 35$

Patel, B., McCully, C., Jha, S. W., et al. 2014, ApJ, 786, 9

Paulin-Henriksson, S., Antonuccio-Delogu, V., Haines, C. P., et al. 2007 A\&A, 467, 427

Peng, E.-H., Andersson, K., Bautz, M. W., \& Garmire, G. P. 2009, ApJ, 701,1283

Planck Collaboration Ade, P. A. R., Aghanim, N., Armitage-Caplan, C., et al. 2014, A\&A, 571, A16

Postman, M., Coe, D., Benítez, N., et al. 2012a, ApJS, 199, 25

Postman, M., Lauer, T. R., Donahue, M., et al. 2012b, ApJ, 756, 159

Prada, F., Klypin, A. A., Cuesta, A. J., Betancort-Rijo, J. E., \& Primack, J. 2012, MNRAS, 423, 3018

Rasia, E., Mazzotta, P., Bourdin, H., et al. 2008, ApJ, 674, 728

Rasia, E., Meneghetti, M., Martino, R., et al. 2012, NJPh, 14, 055018

Rhodes, J., Refregier, A., \& Groth, E. J. 2000, ApJ, 536, 79

Rhodes, J. D., Massey, R. J., Albert, J., et al. 2007, ApJS, 172, 203

Riebe, K., Partl, A. M., Enke, H., et al. 2013, AN, 334, 691

Rosati, P., Borgani, S., \& Norman, C. 2002, ARA\&A, 40, 539 
Sadeh, S., \& Rephaeli, Y. 2008, MNRAS, 388, 1759

Sand, D. J., Treu, T., \& Ellis, R. S. 2002, ApJL, 574, L129

Schmidt, R. W., \& Allen, S. W. 2007, MNRAS, 379, 209

Schneider, P., \& Er, X. 2008, A\&A, 485, 363

Schneider, P., \& Seitz, C. 1995, A\&A, 294, 411

Seitz, S., Schneider, P., \& Bartelmann, M. 1998, A\&A, 337, 325

Sembolini, F., Yepes, G., de Petris, M., et al. 2013, MNRAS, 429, 323

Sereno, M., Giocoli, C., Ettori, S., \& Moscardini, L. 2015, MNRAS, 449, 2024

Sereno, M., Umetsu, K., Ettori, S., \& Baldi, A. 2013, AN, 334, 445

The Dark Energy Survey Collaboration. 2005, arXiv:0510346

Umetsu, K., Broadhurst, T., Zitrin, A., Medezinski, E., \& Hsu, L.-Y. 2011, ApJ, 729, 127

Umetsu, K., Medezinski, E., Broadhurst, T., et al. 2010, ApJ, 714, 1470

Umetsu, K., Medezinski, E., Nonino, M., et al. 2014, ApJ, 795, 163

Umetsu, K., Medezinski, E., Nonino, M., et al. 2012, ApJ, 755, 56 van Waerbeke, L. 2000, MNRAS, 313, 524

Voit, G. M. 2005, RvMP, 77, 207

von der Linden, A., Allen, M. T., Applegate, D. E., et al. 2014, MNRAS, 439,2

Walker, M. G., \& Peñarrubia, J. 2011, ApJ, 742, 20

Wojtak, R., \& Łokas, E. L. 2010, MNRAS, 408, 2442

Zhao, D. H., Jing, Y. P., Mo, H. J., \& Börner, G. 2003, ApJL, 597, L9

Zheng, W., Postman, M., Zitrin, A., et al. 2012, Natur, 489, 406

Zitrin, A., Broadhurst, T., Coe, D., et al. 2011, ApJ, 742, 117

Zitrin, A., Broadhurst, T., Umetsu, K., et al. 2009, MNRAS, 396, 1985

Zitrin, A., Broadhurst, T., Umetsu, K., et al. 2010, MNRAS, 408, 1916

Zitrin, A., Fabris, A., Merten, J., et al. 2014, arXiv:1411.1414

Zitrin, A., Meneghetti, M., Umetsu, K., et al. 2013, ApJL, 762, L30

Zitrin, A., Moustakas, J., Bradley, L., et al. 2012a, ApJL, 747, L9

Zitrin, A., Rosati, P., Nonino, M., et al. 2012b, ApJ, 749, 97 